\title{
Constraints on the alignment of galaxies in galaxy clusters from $\sim 14000$ spectroscopic members ${ }^{\star}$
}

\author{
Cristóbal Sifón ${ }^{1}$, Henk Hoekstra ${ }^{1}$, Marcello Cacciato ${ }^{1}$, Massimo Viola ${ }^{1}$, Fabian Köhlinger ${ }^{1}$, Remco F. J. van der Burg ${ }^{1}$, \\ David J. Sand ${ }^{2}$, and Melissa L. Graham ${ }^{3}$ \\ 1 Leiden Observatory, Leiden University, PO Box 9513, 2300 RA Leiden, The Netherlands \\ e-mail: sifon@strw.leidenuniv.nl \\ 2 Department of Physics, Texas Tech University, 2500 Broadway St., Lubbock, TX 79409, USA \\ 3 Astronomy Department, B-20 Hearst Field Annex \#3411, University of California at Berkeley, Berkeley, CA 94720-3411, USA
}

Received 19 June 2014 / Accepted 25 November 2014

\section{ABSTRACT}

\begin{abstract}
Torques acting on galaxies lead to physical alignments, but the resulting ellipticity correlations are difficult to predict. As they constitute a major contaminant for cosmic shear studies, it is important to constrain the intrinsic alignment signal observationally. We measured the alignments of satellite galaxies within 90 massive galaxy clusters in the redshift range $0.05<z<0.55$ and quantified their impact on the cosmic shear signal. We combined a sample of 38104 galaxies with spectroscopic redshifts with high-quality data from the Canada-France-Hawaii Telescope. We used phase-space information to select 14576 cluster members, 14250 of which have shape measurements and measured three different types of alignment: the radial alignment of satellite galaxies toward the brightest cluster galaxies (BCGs), the common orientations of satellite galaxies and BCGs, and the radial alignments of satellites with each other. Residual systematic effects are much smaller than the statistical uncertainties. We detect no galaxy alignment of any kind out to at least $3 r_{200}$. The signal is consistent with zero for both blue and red galaxies, bright and faint ones, and also for subsamples of clusters based on redshift, dynamical mass, and dynamical state. These conclusions are unchanged if we expand the sample with bright cluster members from the red sequence. We augment our constraints with those from the literature to estimate the importance of the intrinsic alignments of satellites compared to those of central galaxies, for which the alignments are described by the linear alignment model. Comparison of the alignment signals to the expected uncertainties of current surveys such as the Kilo-Degree Survey suggests that the linear alignment model is an adequate treatment of intrinsic alignments, but it is not clear whether this will be the case for larger surveys.
\end{abstract}

Key words. galaxies: clusters: general - galaxies: interactions - gravitational lensing: weak - cosmology: observations

\section{Introduction}

Tidal torques tend to align triaxial satellite galaxies toward the center of the larger "host" gravitational potential as they orbit around its center. This mechanism is well established in numerical simulations, where galaxies are typically locked pointing toward the centers of clusters, possibly with brief periodic misalignments depending on the specific orbit, well within a Hubble time (e.g., Ciotti \& Dutta 1994; Altay et al. 2006; Faltenbacher et al. 2008; Pereira et al. 2008; Pereira \& Bryan 2010). In a hierarchical clustering scenario, this effect could be coupled with alignments arising from the nonlinear evolution of structure. Therefore the patterns and evolution of galaxy alignments - if any - contain important information about the initial conditions that gave rise to the present-day cosmic web, as well as the formation history and environments of galaxies.

Additionally, these galaxy alignments (commonly referred to as "intrinsic," as opposed to apparent, alignments) are a potential contaminant of cosmic shear, which is a measurement of the coherent distortions of galaxies in the background of a matter distribution. While the signal from these intrinsic alignments is weak enough that it is not relevant for weak lensing measurements of galaxy clusters (and in general cluster members can be identified and removed to a sufficient level), it is a concern for large-area cosmic shear surveys, which are more susceptible to this contamination, and where the requirements on precision

$\star$ Table 2 is available in electronic form at http://www . aanda.org and accuracy are more stringent. The contamination induced by these galaxy alignments into cosmic shear measurements can be divided into two effects. The first effect is the tidal alignment of galaxies with similar formation histories, so-called intrinsicintrinsic or II signal. Since this effect is restricted to pairs with common formation or evolutionary histories, this II signal can be avoided by selecting pairs of galaxies with large angular and/or redshift separations (e.g., King \& Schneider 2002; Heymans \& Heavens 2003; Heymans et al. 2004). The second effect is more subtle and more difficult to control: the same gravitational field that aligns galaxies within a halo is responsible for the deflection of the light coming from background galaxies (Hirata \& Seljak 2004). This effect is referred to as gravitational-intrinsic or GI signal (for consistency, the lensing signal itself is referred to as gravitational-gravitational, or GG, signal). In tomographic analyses, it is possible to account for this effect through its distinct redshift dependence (King 2005; Joachimi \& Schneider 2008; Zhang 2010a,b) or, inversely, to measure it from cosmic shear data by boosting its signal while suppressing the contribution from gravitational lensing (Joachimi \& Schneider 2010). This cross-correlation has recently been shown to exist also between galaxy-galaxy lensing and cosmic microwave background lensing (Hall \& Taylor 2014; Troxel \& Ishak 2014). Intrinsic alignments can also be modeled directly in cosmic shear data and marginalized over to extract cosmological parameters (Joachimi \& Bridle 2010; Heymans et al. 2013). In an attempt to identify a consistent model for galaxy shapes and alignments, Joachimi et al. $(2013 \mathrm{a}, \mathrm{b})$ have tried to match semi-analytical models to 
galaxies from the COSMOS survey (Scoville et al. 2007) and find that the intrinsic alignment contamination to upcoming cosmic shear surveys should be $<10 \%$.

Recent large photometric and spectroscopic surveys such as the 2-degree Field redshift survey (2dF; Colless et al. 2001) and the Sloan Digital Sky Survey (SDSS; York et al. 2000) have allowed the study of galaxy alignments out to several tens of Mpc exploiting cross-correlation techniques, with robust direct detections of the GI signal up to $z \sim 0.7$ between galaxy samples with large line-of-sight separations (Mandelbaum et al. 2006; Hirata et al. 2007; Joachimi et al. 2011), although Mandelbaum et al. (2011) reported a null detection. However, the II signal is much weaker than the GI signal in nontomographic studies at intermediate redshifts typical of these surveys, and has typically eluded detection (e.g., Mandelbaum et al. 2006, 2011; Blazek et al. 2012).

On smaller scales the history of these measurements goes back further, but the issue is far from settled. Early measurements of galaxy alignments focused on galaxy clusters, trying to understand galaxy formation and (co-)evolution. Rood \& Sastry (1972) were the first to claim a detection of a preferential direction of galaxies in clusters. Specifically, they found that satellite galaxies in Abell 2199 tend to point in the direction of the major axis of the brightest cluster galaxy (BCG). However, most subsequent measurements have been consistent with random orientations of satellite galaxies in clusters (e.g., Hawley \& Peebles 1975; Thompson 1976; Dekel 1985; van Kampen \& Rhee 1990; Trevese et al. 1992; Panko et al. 2009; Hung \& Ebeling 2012), although some authors have also claimed significant nonrandom orientations of these cluster satellites (e.g., Djorgovski 1983; Godłowski et al. 1998, 2010; Baier et al. 2003; Plionis et al. 2003).

More recent studies have focused on smaller mass galaxy groups, where the number of objects is much larger. Similar to the results summarized above, most of these measurements are consistent with no alignments (Bernstein \& Norberg 2002; Hao et al. 2011; Schneider et al. 2013; Chisari et al. 2014), although there are claims of significant detections (e.g., Pereira \& Kuin 2005; Faltenbacher et al. 2007) ${ }^{1}$. Interestingly, although this effect might be expected to be stronger for more massive halos, Agustsson \& Brainerd (2006) found that satellite galaxies are radially aligned in galaxy-scale halos. However, Hao et al. (2011) and Schneider et al. (2013) have shown that the results can depend on the method used to estimate the direction of the satellite galaxies, so each result must be taken with care.

In general, there is clear tension between observations and numerical N-body simulations, with the latter predicting much higher signals than have been observed. This discrepancy can be attributed, for instance, to a misalignment between stars and dark matter, such that stars - being more centrally concentrated than dark matter - react more slowly and less strongly to tidal torquing from the parent halo (Pereira \& Bryan 2010; Tenneti et al. 2014). Whatever the physical reasons of this discrepancy, the potential impact of the choice of intrinsic alignment model on cosmological parameter estimation (Kirk et al. 2012) makes it imperative that we know the level of intrinsic alignments to high precision at all relevant mass and spatial scales, and this can only be achieved through detailed observations.

In this work, we study the alignments of galaxies in clusters from a sample of galaxy clusters with high-quality photometric observations and a large number of spectroscopic redshifts

\footnotetext{
1 We discuss sources of this discrepancy in light of recent results in Sect. 5.4.
}

from archival sources. We measure different kinds of alignments, assess systematic errors, and use the halo model to characterize galaxy alignments in the context of upcoming cosmic shear analyses.

We adopt a flat $\Lambda$ CDM cosmology with $\Omega_{\Lambda}=0.7, \Omega_{M}=0.3$ and $H_{0}=70 \mathrm{~km} \mathrm{~s}^{-1} \mathrm{Mpc}^{-1}$. To calculate the power spectra, we use $\sigma_{8}=0.8, \Omega_{b} h^{2}=0.0245$, and $n_{s}=1.0$. All magnitudes are MAG_AUTO from SExtractor in the AB system, and all absolute magnitudes and luminosities are in the rest frame of the corresponding cluster.

\section{Data}

\subsection{Cluster sample and photometry}

The cluster sample is drawn from two large, nonoverlapping $\mathrm{X}$-ray selected cluster surveys carried out with the CanadaFrance-Hawaii Telescope (CFHT), namely the Multi-Epoch Nearby Cluster Survey (MENeaCS; Sand et al. 2012) and the Canadian Cluster Comparison Project (CCCP; Hoekstra et al. 2012). MENeaCS performed multi-epoch observations of 57 clusters in the redshift range $0.05<z<0.15$, aimed at measuring the supernova Ia rate in these clusters. For this, clusters were observed using the $g$ and $r$ bands with MegaCam. CCCP was designed to study the scaling relations between different tracers of mass in galaxy clusters, and includes 50 clusters in the redshift range $0.15<z<0.55$. Of these, 20 clusters had archival $B$ - and $R$-band data taken with the CFH12k camera, and 30 clusters were observed with the $g$ and $r$ bands with MegaCam (Hoekstra 2007; Hoekstra et al. 2012).

The data were reduced using the Elixir pipeline (Magnier \& Cuillandre 2004), and processed further following the method outlined in van der Burg et al. (2013), which is summarized below. In order to simplify point spread function (PSF) modeling for shape measurements, we homogenized the PSF across the entire field-of-view by finding a locally-varying convolution kernel that makes the PSF circular and Gaussian everywhere (Kuijken 2008). This PSF homogenization was done for each exposure, after which the individual exposures were co-added. By applying a Gaussian weight function to measure aperture fluxes we optimized color measurements in terms of signal-to-noise ratio (S/N; see Kuijken 2008; and van der Burg et al. 2013, Appendix A). This Gaussianization process introduces correlations in pixel noise, which we do not account for. As we show in Sect. 4.3, this is not a problem for the present analysis.

We performed object detection with SExtractor (Bertin \& Arnouts 1996) in dual-image mode, using the $r$ (or $R$ ) band as detection image. All sources were detected in the $r$-band image obtained by stacking the nonhomogenized images. Photometric zero points were calibrated using the stellar locus regression (SLR) software developed by Kelly et al. (2014) ${ }^{2}$. SLR uses the known colors of stars to obtain solutions for the photometric zero points of any photometric catalog, correcting for instrumental response and atmospheric and Galactic extinctions (see also High et al. 2009). We used the Two-Micron All Sky Survey (2MASS; Skrutskie et al. 2006) $J$-band star catalog in addition to our MegaCam $g$ and $r$, or CFH12k $B$ and $R$, observations as inputs to the SLR. We retrieved extinction values in the $J$ band from the NASA/IPAC Extragalactic Database (NED) ${ }^{3}$, which use the reddening measurements of Schlafly \& Finkbeiner (2011). With these two colors, plus the absolute photometric

\footnotetext{
2 https://code.google.com/p/big-macs-calibrate/

3 http://ned.ipac.caltech.edu/
} 
Table 1. Spectroscopic catalogs.

\begin{tabular}{|c|c|c|c|c|}
\hline Source & $\begin{array}{c}\text { Total } \\
\text { redshifts }\end{array}$ & $\begin{array}{l}\text { Unique } \\
\text { redshifts }\end{array}$ & Clusters & $\begin{array}{l}\text { Avg. unique } \\
\text { per cluster }\end{array}$ \\
\hline \multicolumn{5}{|c|}{ Compilations } \\
\hline NED & 16125 & 9161 & 79 & 116 \\
\hline WLTV & 1613 & 1399 & 2 & 700 \\
\hline $\mathrm{CNOC}$ & 1427 & 1266 & 10 & 127 \\
\hline SDSS DR10 & 14634 & 13995 & 62 & 226 \\
\hline $\mathrm{HeCS}$ & 8470 & 8368 & 27 & 310 \\
\hline MENeaCS-spec & 1966 & 1966 & 12 & 164 \\
\hline \multicolumn{5}{|c|}{ Single-cluster } \\
\hline Geller et al. (2014) & & 834 & 1 & 834 \\
\hline Ebeling et al. (2014) & & 1115 & 1 & 1115 \\
\hline Total & & 38104 & 90 & 423 \\
\hline
\end{tabular}

calibrations of 2MASS (including the extinction correction), we obtained absolute zero points for the CFHT catalogs. For clusters within the SDSS footprint we also use the SDSS griz photometry to check for consistency, from which we conclude that SLRcorrected zero points are calibrated to an absolute uncertainty of $\sim 0.01$ mag. Galaxies were separated from stars by visual inspection of the magnitude-size ${ }^{4}$ plane for each cluster individually. Stars occupy a well-defined region in this plane, having essentially a single size up to the saturation flux. Given that the stacks generally have a sub-arcsecond sized PSF, galaxies used here are large compared to the PSF and are therefore easily distinguishable from stars.

We computed $r$-band absolute magnitudes, $M_{r}$, using $\mathrm{EzGal}^{5}$ (Mancone \& González 2012), using a passive evolution Charlot \& Bruzual 2007 (unpublished, see Bruzual \& Charlot 2003) single burst model with solar metallicity and formation redshift $z_{f}=5$.

\subsection{Spectroscopic data}

We searched for spectroscopic redshifts around all clusters in the MENeaCS+CCCP sample in six archival sources: NED, the WIYN Long-Term Variability survey (WLTV; Crawford et al. 2011), the Canadian Network for Observational Cosmology Survey (CNOC; Yee et al. 1996, 1998; Ellingson et al. 1997; Abraham et al. 1998), the SDSS data release $10^{6}$ (DR10; Ahn et al. 2014) which is part of SDSS-III (SDSS-III Collaboration 2012) and the Hectospec Cluster Survey (HeCS; Rines et al. 2013). We also include redshifts from the MENeaCS spectroscopic survey (hereafter MENeaCS-spec). When a galaxy was found in more than one of these catalogs, each catalog replaced the preceding as listed above and in Table 1. Thus we included all redshifts from MENeaCS-spec. In NED and in SDSS DR10, we searched for galaxies with spectroscopic redshifts within a radius of 1 deg around each cluster, but only galaxies in our photometric catalogs were included in the analysis. From NED, we discarded all flagged redshifts (i.e., all those whose Redshift Flag field is not blank) and kept only redshifts with at least 4 significant digits to ensure that only spectroscopic redshifts with enough precision are included. From SDSS we only included galaxies with zWarning $=0$. The NED search includes redshifts obtained as part of large surveys such as the $2 \mathrm{dF}$, the 2MASS spectroscopic survey (2MRS), the WIde-field Nearby

\footnotetext{
4 Here, sizes are given by FLUX_RADIUS from SExtractor.

5 http://www . baryons.org/ezgal/

6 http://www.sdss3.org/dr10/data_access/
}

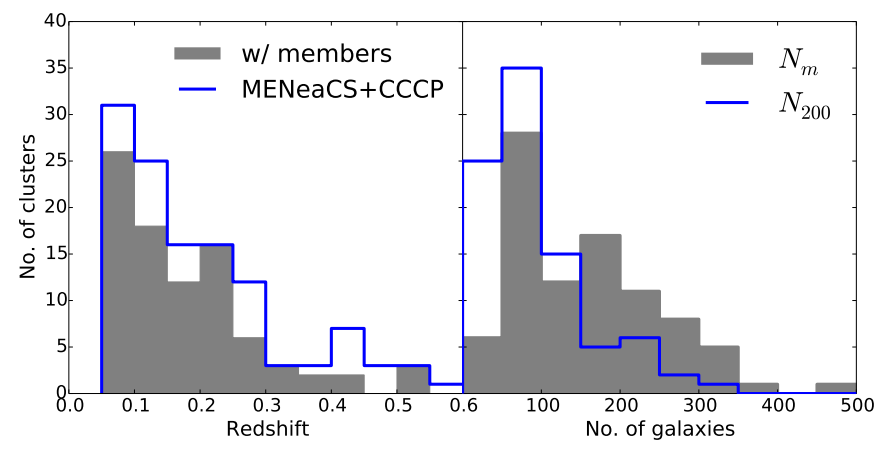

Fig. 1. Left: redshift distributions of all MENeaCS+CCCP clusters (blue histogram) and clusters used in this study (gray filled histogram). Right: distributions of number of spectroscopic members, $N_{m}$ (gray filled histogram), and number of spectroscopic members within $r_{200}, N_{200}$ (blue histogram). Abell 2142, with $N_{m}=1052$ and $N_{200}=731$, is not shown.

\section{Galaxy cluster Survey (WINGS), and the WiggleZ Dark Energy Survey.}

Additionally, we included the redshift catalogs of Abell 383 and MACS J0717.5+3745 recently published by Geller et al. (2014) and Ebeling et al. (2014), respectively, which total 1949 redshifts within our CFHT images. From the catalog of Ebeling et al. (2014) we used only redshifts with quality flag 1 or 2 . We also highlight the redshift catalog of Abell 2142 by Owers et al. (2011), containing $\sim 1800$ galaxies, which is included as part of the NED catalog.

Table 1 lists the number of (unique) spectroscopic redshifts included from each catalog and from how many cluster fields they are taken. The largest redshift catalog(s) for each cluster are listed in Table 2, including the largest catalogs within NED; for most clusters the NED redshifts come mainly from one or two large catalogs (with $\gtrsim 90 \%$ of redshifts). The final spectroscopic sample is summarized in the last row of Table 1: it contains 38104 redshifts in the direction of 90 clusters, selected to have at least 30 members, at least 10 of which must be within $r_{200}$ (see Sect. 3.1). The left panel of Fig. 1 shows the redshift distribution of these 90 clusters, compared to the entire ME$\mathrm{NeaCS}+\mathrm{CCCP}$ sample. The analysis in this paper refers only to these 90 clusters, which are listed in Table 2.

\section{Galaxy samples}

\subsection{Spectroscopic members and dynamical masses}

Spectroscopic membership is determined using the shifting gapper method (Fadda et al. 1996), slightly adjusted from the implementation of Sifón et al. (2013). We bin galaxies in radial bins of at least 15 galaxies and $250 \mathrm{kpc}$ and, for each radial bin, members are selected as those galaxies that are within $800 \mathrm{~km} \mathrm{~s}^{-1}$ from the main body of galaxies, which is bound by gaps of $400 \mathrm{~km} \mathrm{~s}^{-1}$. The reduction in the velocity gaps compared to Sifón et al. (2013) - who used 1000 and $500 \mathrm{~km} \mathrm{~s}^{-1}$, respectively - is due to the larger number of galaxies used here, producing a distribution that has less obvious gaps in velocity space. In some cases, we introduced a radial cut determined from visual inspection. The left panel of Fig. 2 shows that the distribution of confirmed cluster members is similar at low and high redshift for luminous $\left(M_{r} \lesssim-21\right)$ galaxies but low-luminosity galaxies used here come mainly from low redshift clusters. We iteratively measure the velocity dispersion, $\sigma_{200}$, as the biweight estimator of scale (Beers et al. 1990) using all galaxies within $r_{200}$, correcting for measurement errors (Danese et al. 1980). Since measurement 


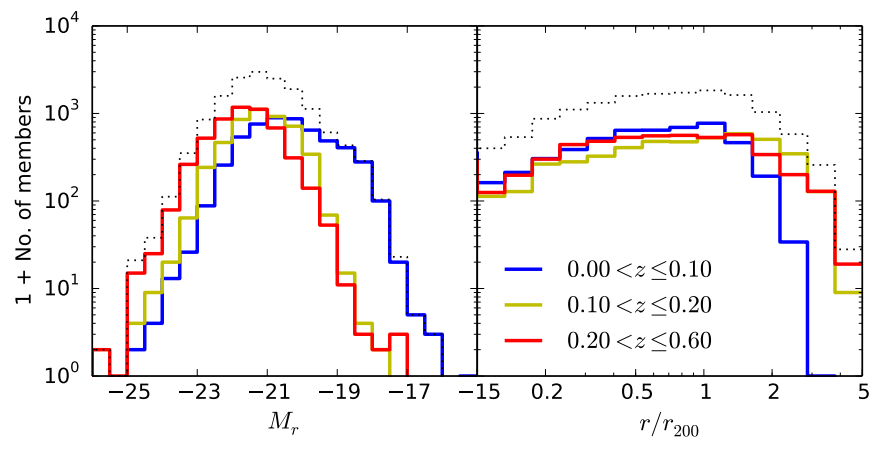

Fig. 2. Distribution of spectroscopic members. Left: as a function of rest-frame absolute $r$-band magnitude. Right: as a function of clustercentric distance in units of $r_{200}$. The dotted black lines show the distribution of the full sample; the solid lines show the distribution split into three redshift bins of approximately equal number of clusters.

uncertainties are not available for all galaxies (most notably, they are not given in NED), we use a fiducial value of $150 \mathrm{~km} \mathrm{~s}^{-1}$ for the uncertainty of all redshifts, which is a conservative estimate for recent measurements, but can be representative of older or low resolution measurements listed in NED. The change in mass introduced by this correction is, in any case, at the percent level for a cluster with $\sigma \sim 1000 \mathrm{~km} \mathrm{~s}^{-1}$. The cluster redshift is determined iteratively in this process as the biweight estimator of location, considering again galaxies within $r_{200}$. We estimate the mass within $r_{200}$, namely $M_{200}$, using the $\sigma_{200}-M_{200}$ relation of Evrard et al. (2008), and estimate $r_{200}=\left[3 /(4 \pi) \cdot M_{200} /\left(200 \rho_{c}\right)\right]^{1 / 3}$. The resulting $\sigma_{200}$ is insensitive to uncertainties in $r_{200}$; varying $r_{200}$ by $\pm 20 \%$ from those obtained from this relation only changes $\sigma_{200}$ by $\$ 5 \%$ for every cluster (in other words, velocity dispersion profiles are very close to flat near $r_{200}$ ). The uncertainties in the velocity dispersion are obtained from 1000 jackknife samples drawn from all galaxies with peculiar velocities up to 3 times the cluster velocity dispersion; therefore quoted uncertainties include an estimate of the effect of membership selection. Uncertainties in the dynamical mass are obtained by propagating the uncertainties on the velocity dispersion. The dynamical properties described above are listed in Table 2, together with the number of members, $N_{m}$, and the number of members within $r_{200}, N_{200}$. The right panel of Fig. 1 shows the distribution of $N_{200}$ and $N_{m}$, the number of members out to arbitrary radius.

In cases where the spectroscopic members do not reach out to $r_{200}$, we cannot infer $\sigma_{200}$ directly from the data. We therefore apply a correction to the measured velocity dispersion assuming the isotropic velocity dispersion profile of Mamon et al. (2010) and the mass-concentration relation of Duffy et al. (2008) to get the theoretical expectation for $\sigma_{200}$ given $\sigma\left(<r_{\max }\right)$. This correction is linear with $r_{\max }$ for $r_{\max } \gtrsim 0.2 r_{200}$, with correction factors 0.93 and 0.81 for the velocity dispersion and mass, respectively, for $r_{\max }=0.6 r_{200}$ (i.e., the mass within $0.6 r_{200}$ is $0.81 M_{200}$ ), only weakly dependent on mass and redshift. In our sample there are 14 clusters with $r_{\max }<r_{200}$, with a median of $r_{\max } / r_{200}=0.69$ and 10th and 90th percentiles of 0.51 and 0.79 , respectively. For these clusters, we list the corrected values in Table 2.

There are a total of 14576 cluster members among 90 clusters, 9054 of which are within $r_{200}$. The radial distribution of cluster members is shown in the right panel of Fig. 2. The spectroscopic members on average follow a Navarro-FrenkWhite (NFW; Navarro et al. 1995) profile with concentration $c_{200} \sim 2$ (van der Burg et al. 2015). The median redshift of

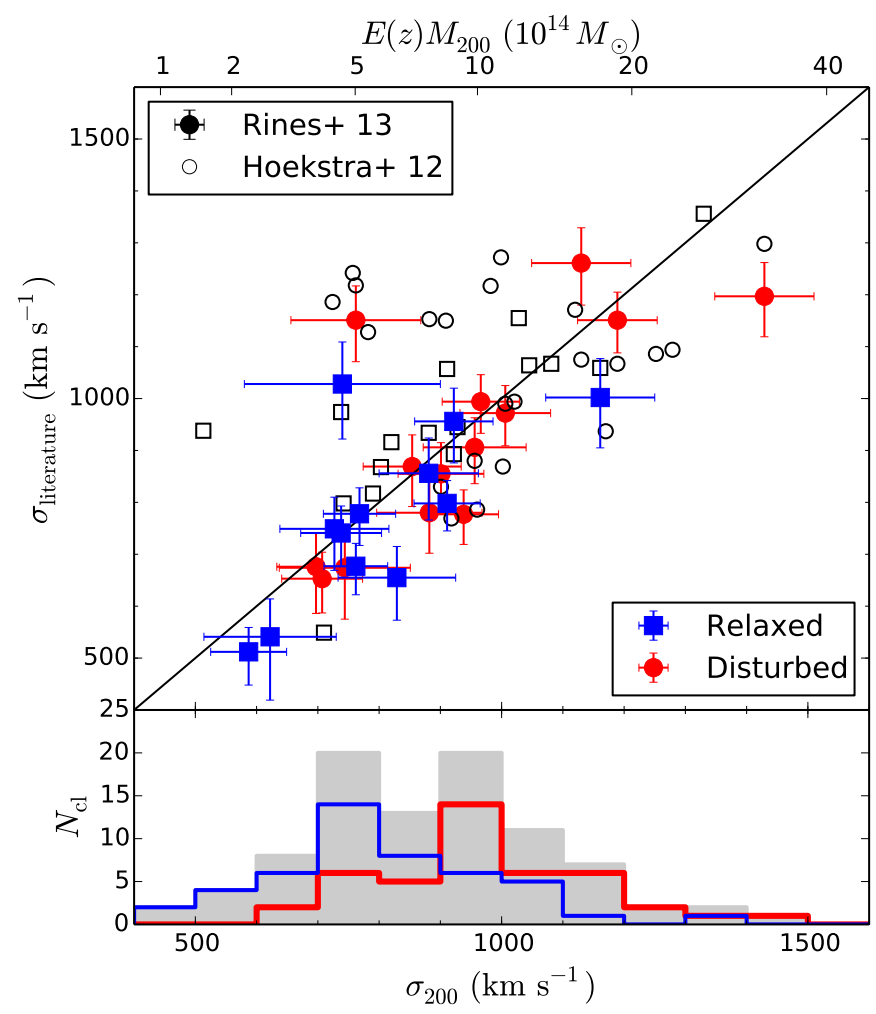

Fig. 3. Top: comparison between velocity dispersions calculated from spectroscopic members in this work with those in Rines et al. (2013), and with velocity dispersions calculated by fitting a single isothermal sphere to the weak lensing profile (Hoekstra et al. 2012) for CCCP clusters used in this work. Errorbars are not shown for the latter for clarity. Squares and circles show relaxed and disturbed clusters, respectively. The black line shows the one-to-one relation, and the top axis shows $E(z) M_{200}$ for a given $\sigma_{200}$ from the Evrard et al. (2008) relation. Bottom: distribution of velocity dispersions of the full sample. The gray histogram shows the total distribution, with the blue and red histograms showing the distributions for relaxed and disturbed clusters, respectively.

the sample is $z=0.144$, and the median velocity dispersion is $\sigma_{200}=881 \mathrm{~km} \mathrm{~s}^{-1}$ which translates to a median dynamical mass $M_{200}=7.2 \times 10^{14} M_{\odot}$ and a median size $r_{200} \sim 1.7 \mathrm{Mpc}$. The distribution of $\sigma_{200}$ is shown in the bottom panel of Fig. 3 .

In Fig. 3, we compare the present velocity dispersions to those of Rines et al. (2013); there is a large overlap between the two data sets (see Table 1). The two sets of measurements are consistent, with a median ratio $\left\langle\sigma_{200} / \sigma_{\text {Rines }+13}\right\rangle=1.04 \pm 0.03$. We also show, for comparison, the singular isothermal sphere velocity dispersions fit by Hoekstra et al. (2012) to the weak lensing signal of 39 overlapping clusters, which are also consistent with our measurements to within $2 \%$ on average. It is apparent from Fig. 3 that the agreement between $\sigma_{200}$ and $\sigma_{\mathrm{WL}}$ is better for relaxed clusters than for disturbed ones, consistent with expectations. For comparison, using the velocity gaps used by Sifón et al. (2013) in our analysis, we obtain velocity dispersions larger than those of Rines et al. (2013) by $\sim 14 \%$.

\subsubsection{Dynamical state}

We can take further advantage of our large spectroscopic catalogs by studying the dynamical states of clusters. To this end we use the DS test (Dressler \& Schechtman 1988), which uses both the positions and velocities of galaxies. The DS test gives a 

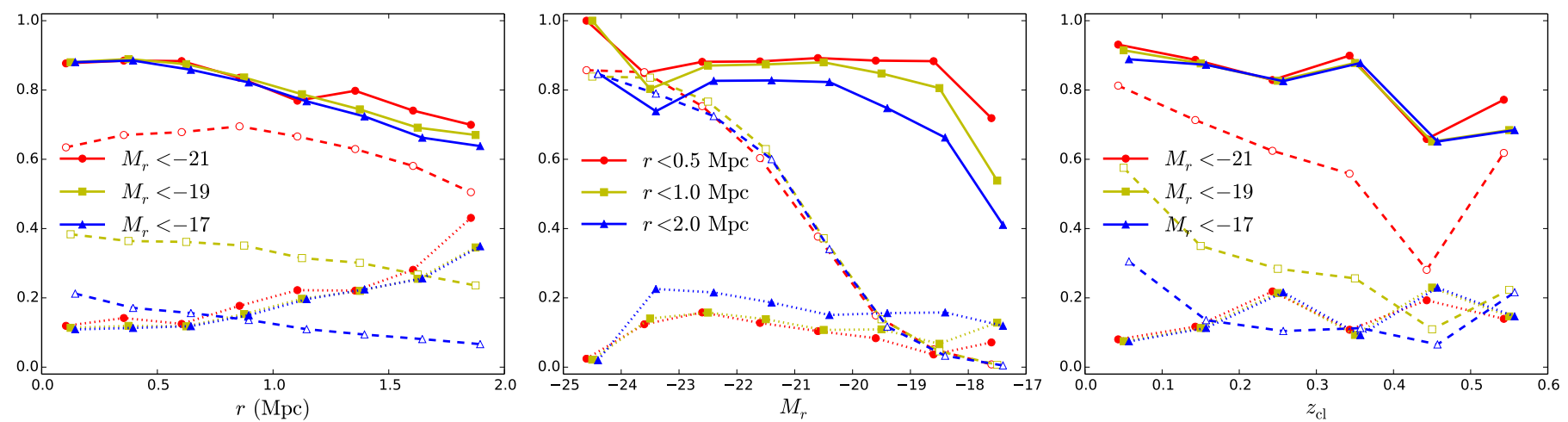

$\longrightarrow N_{\mathrm{RS}+\mathrm{spec}+\text { member }} / N_{\mathrm{RS}+\mathrm{spe}}$

- o- $N_{\mathrm{RS}+\text { spec }} / N_{\mathrm{RS}}$

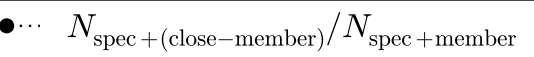

Fig. 4. Purity of the red sequence (filled symbols with solid lines) and spectroscopic completeness within the red sequence (open symbols with dashed lines). Filled symbols with dotted lines show the fraction of galaxies that are not selected as members but that are within $\Delta z=0.03(1+z)$ of the cluster, which represents the contamination in an unbiased photometric redshift selection. Left: as a function of cluster-centric distance, for different luminosity limits. Middle: as a function of absolute magnitude, for different radial apertures. Right: as a function of redshift for different luminosity limits, at an aperture of $1 \mathrm{Mpc}$. Note that points within a given line are independent (each line is a differential distribution), but lines of the same type are not independent from each other.

measure of substructure by identifying galaxies that do not follow the cluster velocity distribution through the metric

$\delta^{2}=\frac{N_{\text {local }}}{\sigma^{2}}\left[\left(\bar{v}_{\text {local }}-\bar{v}\right)^{2}+\left(\sigma_{\text {local }}-\sigma\right)^{2}\right]^{2}$,

where $\bar{v}_{\text {local }}$ and $\sigma_{\text {local }}$ are the local velocity and velocity dispersion, measured for the $N_{\text {local }}$ nearest neighbors around a test member, and $\bar{v}$ and $\sigma$ are the global values. The $\Delta$-statistic is the sum of $\delta$ 's over all cluster members. This statistic is then measured 5000 times after shuffling the velocities of cluster members, keeping positions fixed, with the same $N_{\text {local }}$. The substructure significance (hereafter $\mathcal{S}_{\Delta}$ ) is the fraction of random samples which have $\Delta$ higher than that of the cluster. Errorbars are $68 \%$ ranges obtained from 5 runs for each cluster, varying the number of neighbors within $\sqrt{N_{200}}-2 \leq N_{\text {local }} \leq \sqrt{N_{200}}+2$, and the central value is their median. We run the DS test using only members within $r_{200}$ because $r_{200}$ is very close to the virial radius, beyond which the cluster should not be relaxed, by definition.

The DS test is not designed to assess the dynamical state of clusters in general but specifically to find substructure, which furthermore has to have a different spatial and velocity location from the cluster itself. It is therefore incomplete; there are indeed examples of known merging clusters from which the DS test cannot find indications of substructure, most notably mergers along the plane of the sky (e.g., Menanteau et al. 2012; Barrena et al. 2013). This is the case here with Abell 520 (e.g., Jee et al. 2014), for example. By means of $N$-body simulations, Pinkney et al. (1996) showed that $\mathcal{S}_{\Delta}<0.05$ is a reasonable condition to define a pure, but not necessarily complete, sample of dynamically disturbed clusters. We follow the results of Pinkney et al. (1996) in a conservative way, selecting as disturbed all clusters that are consistent with $\mathcal{S}_{\Delta} \leq 0.05$ within errorbars (52 clusters). All others are classified as relaxed ( 38 clusters). This is conservative in the sense that we aim to construct a pure sample of relaxed clusters (see Sect. 5.1). Table 2 lists $\mathcal{S}_{\Delta}$ together with the classification for each cluster. We find that more massive clusters tend to be classified as disturbed (D), while less massive clusters are generally relaxed (R); this can be seen in Fig. 3.

\subsection{Red sequence members}

While spectroscopy provides a clean sample of member galaxies from precise velocities, it suffers from incompleteness mainly due to two practical reasons: 1) obtaining a redshift for a galaxy is expensive; typically it takes $\sim 30$ min of observations for galaxies in low-redshift $(z \lesssim 0.5)$ clusters, depending on the telescope and observing conditions; and 2) only a limited number of galaxies can be targeted in a single observation because of slit overlap or fiber collisions.

Being a distinct feature of clusters, the red sequence provides an ideal complement to spectroscopic members. As we show below, for luminous galaxies near the centers of clusters this also provides a clean membership selection, though not as clean as spectroscopy. Using the red sequence in addition to the spectroscopic selection ensures that only a small fraction of galaxies need to be included through this more uncertain method, making the purity of the sample very close to $100 \%$.

To find the red sequence in each cluster, we first separate blue and red galaxies by fitting two one-dimensional Gaussians to the color distribution of galaxies using an Error-Corrected Gaussian Mixture Model (ECGMM; Hao et al. 2009). We then fit a straight line in color-magnitude space to the red galaxies using a maximum likelihood approach that accounts for intrinsic scatter and the measurement uncertainty in color, iteratively rejecting $2 \sigma$ outliers. Details will be presented in a forthcoming paper (Sifón et al., in prep.).

We assess the purity of the red sequence as a cluster member selection procedure by looking at red sequence members that have redshifts. There are in total 57885 red sequence galaxies up to $M_{r}=-17$ and within $2 \mathrm{Mpc}, 7224$ of which have a redshift measurement $(\sim 12 \%)$. Figure 4 shows that the red sequence is a high-fidelity member selection method even to large radii. Only the sample of both low-luminosity $\left(M_{r} \gtrsim-19\right)$ and distant $(r \gtrsim 1 \mathrm{Mpc}$ ) red sequence members has a lower purity, although the latter is still $\gtrsim 70 \%$ for most of this distance-luminosity space. We include in the extended sample all red sequence galaxies more luminous than $M_{r}=-19$ within $1 \mathrm{Mpc}$ of the cluster center. Within these parameter boundaries, $84 \%$ of red sequence galaxies with a spectroscopic redshift are confirmed 


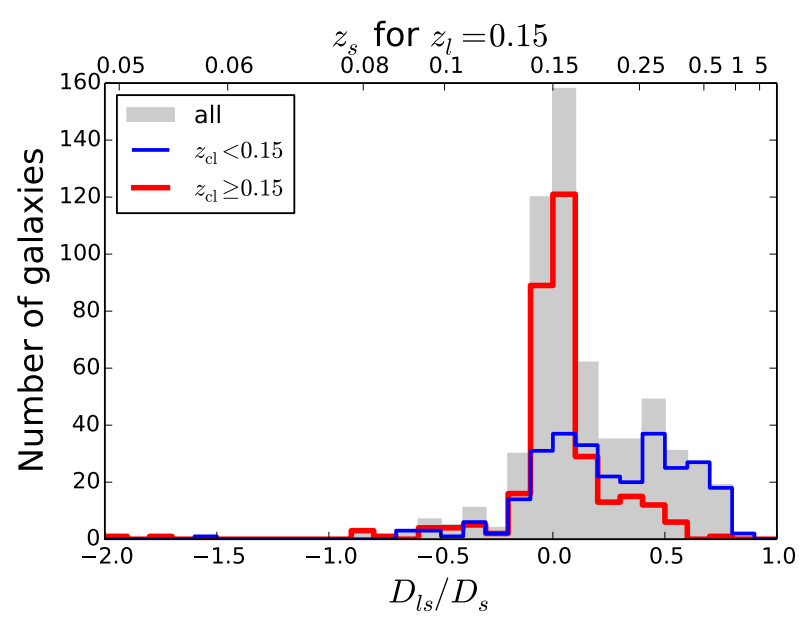

Fig. 5. Distribution of the distance ratio, $D_{\mathrm{ls}} / D_{\mathrm{s}}$, for red sequence members that are confirmed to be nonmembers of the clusters from spectroscopic redshifts. The gray filled histogram shows red sequence galaxies from all clusters; the blue and red (empty) histograms show the distributions for clusters at low and high redshift, respectively. For illustration, the top axis shows the source redshift for a cluster at $z=0.15$.

cluster members. This level of contamination ( $16 \%$ of red sequence members) has no effects on our results.

The rightmost panel of Fig. 4 shows that up to $z \sim 0.4$, the purity is extremely high $(\sim 90 \%)$ but then decreases to $\sim 70 \%$, because above $z \sim 0.4$ the $4000 \AA$ break is no longer bracketed by the $g$ and $r$ bands (similarly for the $B$ and $R$ bands). The completeness of the spectroscopic samples does drop noticeably with redshift because of the higher difficulty posed by spectroscopic observations of high redshift clusters.

From a lensing perspective, one other important ingredient in assessing the red sequence is the redshift distribution of the contaminating fraction, which we can quantify using red sequence galaxies that are confirmed to be outside the cluster. If they are sufficiently far behind the cluster, they could in fact be lensed, inducing a signal that we wish to avoid. If instead they are either very close behind or in front of the cluster, then they will not be lensed and will only dilute the signal. Figure 5 shows the redshift distribution of red sequence galaxies that are confirmed to be nonmembers through the distance ratio, $D_{\mathrm{ls}} / D_{\mathrm{s}}$, where $D_{\mathrm{ls}}$ is the angular diameter distance between the lens (i.e., cluster) and the source galaxy, and $D_{\mathrm{s}}$ is the angular diameter distance to the source. Note that $D_{\mathrm{ls}}<0$ for $z_{\mathrm{s}}<z_{\mathrm{l}}$. Galaxies behind the clusters are lensed, resulting in (apparent) tangential alignments. The amplitude of this effect can be quantified by the "lensing efficiency", $\beta$, defined as

$\beta \equiv\left\langle\max \left(0, \frac{D_{\mathrm{ls}}}{D_{\mathrm{s}}}\right)\right\rangle$,

which is typically calculated using photometric redshifts or an average redshift distribution. Note that Eq. (2) naturally accounts for galaxies in front of the cluster, which do not contain a lensing signal (but do introduce noise), which is especially important when using a generic redshift distribution, or full photometric redshift probability distributions (in which case background galaxies have a nonzero probability of being in front of the cluster). Of the 688 confirmed nonmembers in the red sequence, 496 (72\%) are behind the cluster (incuding those immediately behind the cluster), and the lensing efficiency of red sequence nonmembers is $\beta=0.085$. It is therefore possible that the contaminating red sequence galaxies contain some lensing signal from background galaxies, but within the red sequence selection limits imposed here, this sample is only $16 \%$ of the red sequence galaxies. Therefore there is a fraction $\sim 0.72 \times 0.16 \simeq 12 \%$ of contaminating galaxies (with $\sim 0.28 \times 0.16 \simeq 4 \%$ - those in the foreground - adding noise). The lensing signal in these galaxies is $\gamma_{+, \mathrm{rs}} \lesssim 0.11 \cdot \beta \cdot \gamma_{+}=0.11 \cdot 0.085 \cdot 0.10 \approx 9 \times 10^{-4}$, several times smaller than the statistical uncertainties (where $\gamma_{+} \approx 0.1$ is a typical shear amplitude in the inner regions of galaxy clusters).

In summary, the red sequence gives a high-fidelity cluster member selection. It is important, however, to restrict this selection to the inner regions of clusters and to luminous galaxies (as shown in Fig. 4), because the red sequence may contain some lensing signal. The purity of the red sequence as selected here is $84 \%$, so this contamination is not expected to be significant. Adding the red sequence members to the 14576 spectroscopically confirmed members gives a total of $N_{m}+N_{\mathrm{rs}}=23041$ members with an estimated contamination of $0.16 \cdot N_{\mathrm{rs}} /\left(N_{m}+N_{\mathrm{rs}}\right) \approx 8 \%$.

\subsection{Photometric redshift contamination}

By taking a fixed width in velocity, we can simulate the members found by an accurate, unbiased photometric redshift criterion. The dotted lines in Fig. 4 show the fraction of galaxies that are within $\Delta z=0.03(1+z)$ (as expected for large ongoing photometric redshifts) but are not members of the cluster ${ }^{7}$, as determined in Sect. 3.1. The contamination is roughly independent of magnitude at all radii and at a level of $\sim 13 \%$ within $1 \mathrm{Mpc}$ of the BCG, rising steeply beyond this radius. In terms of apparent magnitude the curves look similar in the range $m_{r} \lesssim 23$, the range in which most of the selected red sequence galaxies are found. This contamination rises shallowly with redshift, reaching $\sim 20 \%$ at $z \gtrsim 0.3$.

The radial dependence in Fig. 4 is shown in physical units instead of in units of $r_{200}$ because this is more generally used with photometric surveys where the physical size of each cluster is not known, and Fig. 4 gives an idea of the apertures that should be used to either search for clusters or characterize the cluster based on a red sequence sample.

Comparing the dotted and solid curves, it seems that there is not such a significant gain in using photo- $z$ 's versus the red sequence. A photo- $z$ selection has the advantage that it selects a more representative population of the cluster, and that the red sequence depends on a single color (at least in this implementation) and it becomes less reliable when the $4000 \AA$ break is not bracketed by the filters used. This is the case in our study for $z \gtrsim 0.36$. It is also apparent, as with the red sequence, that a photo- $z$ selection becomes significantly contaminated beyond $r \sim 1 \mathrm{Mpc}$.

Finally, we note that the galaxies we refer to here (shown with the dotted lines) are not cluster members but also do not contribute a lensing signal, because they are too close behind. They are, indeed, likely to be part of the same large-scale structure of the cluster so would probably feel tidal torque from it similar to the actual member galaxies. Thus from the perspective of galaxy alignment measurements these galaxies should not dilute the signal significantly, nor introduce a lensing signal.

\footnotetext{
$7 \Delta z=0.03(1+z)$ corresponds to $\approx 10000 \mathrm{~km} \mathrm{~s}^{-1}$ at the median redshift of the sample, $z=0.15$.
} 


\subsection{Control samples}

We construct two catalogs as control samples to assess spurious contributions to our measurements. The shapes of objects in these two samples are unaffected by the cluster (and are mostly unrelated between objects in each sample), so their alignment signals (see Sect. 4) should be consistent with zero. A departure from zero would mean that there is significant residual PSF ellipticity in the images, and therefore that the shape measurements are unreliable.

First, we use all stars in the magnitude range $17<m_{r}<22$, selected as outlined in Sect. 2.1, for a total of 443321 stars. We choose the bright limit to avoid saturated stars, whereas the faint limit ensures that the star sample is not contaminated by faint, unresolved galaxies.

We also use all spectroscopically confirmed foreground galaxies, which are selected as all galaxies with peculiar velocities more negative than $-10000 \mathrm{~km} \mathrm{~s}^{-1}$ in the rest-frame of the cluster. There are 3666 spectroscopically confirmed foreground galaxies in the direction of 73 clusters. The clusters with the most foreground galaxies are two of the highest-redshift clusters of the sample, namely MS 0451.6-0305 at $z=0.538$ and MACS J0717.5+3745 at $z=0.544$, with $N_{\mathrm{fg}}=306$ and $N_{\text {fg }}=304$, respectively.

\section{Measuring intrinsic alignments}

We measure the alignment signal of galaxies within clusters by weight-averaging the ellipticity components of all galaxies within a given radial annulus,

$\left\langle\epsilon_{i}\right\rangle=\frac{\sum_{n} w_{n} \epsilon_{i, n}}{\sum_{n} w_{n}}$

with weights equal to

$w_{n}=\frac{1}{\epsilon_{\text {int }}^{2}+\sigma_{n}^{2}}$,

where $\sigma_{n}$ is the measurement uncertainty on the ellipticity of the $n$th galaxy. We assume an intrinsic (i.e., unlensed) galaxy ellipticity dispersion $\epsilon_{\text {int }}=\sqrt{\left\langle\epsilon_{i} \epsilon_{i}\right\rangle}=0.25$. The uncertainty in Eq. (3) is equal for both components and is given by $\sigma\left(\epsilon_{i}\right)=$ $\left(\sum_{n} w_{n}\right)^{-1 / 2}$. In this work, we use the shapes of cluster members to measure three kinds of alignment: the alignment of (satellite) galaxies toward the center of the cluster, the alignment of galaxies with respect to the BCG orientation, and the alignment between satellite galaxies. These three quantities are detailed below.

Throughout, we refer to raw ellipticities as $e_{i}$, and to ellipticities that account for instrumental effects (i.e., PSF size in the case of Gaussianized images) as $\epsilon_{i}$.

\subsection{Different alignment signals}

In this section we outline the different rotations we apply to the ellipticity measurements of Sect. 4.2 in order to extract alignment signals within clusters.

\subsubsection{Satellite radial alignment}

We measure the alignment of galaxies with respect to the center of the cluster using ellipticity components rotated to a frame such that

$\epsilon_{+}=-\left(\epsilon_{1} \cos 2 \theta+\epsilon_{2} \sin 2 \theta\right)$,

$\epsilon_{\mathrm{X}}=\epsilon_{1} \sin 2 \theta-\epsilon_{2} \cos 2 \theta$,

where $\epsilon_{1}$ and $\epsilon_{2}$ are the galaxy ellipticities in the Cartesian frame, with $\epsilon_{1}$ measuring the ellipticity in the $x$ and $y$ directions, and $\epsilon_{2}$ in diagonal directions. Here $\theta$ is the azimuthal angle with respect to the center of the cluster. In this frame, $\epsilon_{+}$measures the distortion in the tangential and radial directions while $\epsilon_{\times}$measures the distortion at $\pm 45^{\circ}$ from the radial direction (see, e.g., Fig. 1 of Bernstein \& Norberg 2002, for a diagram). Note that the definition of $\epsilon_{+}$in Eq. (5) has the opposite sign to that typically used in weak lensing analyses. For symmetry reasons the cross component, $\left\langle\epsilon_{\times}\right\rangle$, of an ensemble of clusters should be consistent with zero (although a single cluster might have a preferred nonradial alignment direction such that $\left\langle\epsilon_{\times}\right\rangle \neq 0$, the average over an ensemble of clusters must be zero), so it serves as a check for systematic effects. On the other hand, $\left\langle\epsilon_{+}\right\rangle\langle 0$ indicates that galaxies are preferentially aligned in the tangential direction, as is the case for gravitationally lensed background galaxies, while $\left\langle\epsilon_{+}\right\rangle>0$ would indicate a radial alignment of the galaxies, which could be the case for cluster members. Finally, $\left\langle\epsilon_{+}\right\rangle=0 \mathrm{im}-$ plies that galaxies are randomly oriented toward the center of the cluster.

\subsubsection{Satellite-BCG alignment}

To measure the alignment between satellite galaxies and the $\mathrm{BCG}$, we rotate the shapes and coordinates of satellites to a frame where the direction of $\epsilon_{1}>0$ coincides with the major axis of the BCG, namely

$\epsilon_{1}^{\prime}=\epsilon \cos \left[2\left(\phi-\phi_{\mathrm{BCG}}\right)\right]$

$\epsilon_{2}^{\prime}=\epsilon \sin \left[2\left(\phi-\phi_{\mathrm{BCG}}\right)\right]$,

where $\phi$ and $\phi_{\mathrm{BCG}}$ are the position angles of a satellite galaxy and the BCG, respectively, and $\epsilon \equiv\left(\epsilon_{1}^{2}+\epsilon_{2}^{2}\right)^{1 / 2}$ is the ellipticity of the galaxy. In this new frame, the BCG has ellipticity components $\epsilon_{1}^{\prime}=\epsilon$ and $\epsilon_{2}^{\prime}=0$. For the BCG position angles we use only GALFIT measurements (see Sect. 4.2.2), since these are expected to be more reliable for galaxies as large as BCGs. Analogous to the radial alignments, $\left\langle\epsilon_{1}^{\prime}\right\rangle>0$ implies that satellite galaxies are oriented along the major axis of the BCG, $\left\langle\epsilon_{1}^{\prime}\right\rangle<0$ that satellites are oriented along the BCG minor axis, and $\left\langle\epsilon_{1}^{\prime}\right\rangle=0$ implies random orientations; $\epsilon_{2}^{\prime}$ measures diagonal alignments so we expect $\left\langle\epsilon_{2}^{\prime}\right\rangle=0$.

\subsubsection{Satellite-satellite alignment}

Finally, we compute the alignment between satellite galaxies within clusters by calculating Eq. (5) taking every satellite galaxy as a test galaxy (i.e., as the frame for $\theta$ ). BCGs are excluded from this analysis. This probes potential alignments of galaxies in substructures within the cluster. In principle, if there are $N$ members in a cluster, the number of pairs is equal to $N(N-1) / 2$. However, we only use pairs for which a full circle can be averaged, to avoid averages that include mostly objects in the corners of the images where PSF residuals may be larger. This is a concern for massive, low redshift clusters, where $30^{\prime}$ (half the side of the MegaCam image) is roughly equal to $r_{200}$ in the worst cases. To ensure that the average remains unbiased, therefore, we only include pairs such that the sum of the distance between the test galaxy and the center of the cluster and the separation between the two satellites is less than $90 \%$ of the distance between the cluster center and the edge of the image. 


\subsection{Shape measurements}

Measuring galaxy shapes is a challenging endeavor, especially in the presence of noise and PSF anisotropies (e.g., Massey et al. 2007; Melchior \& Viola 2012; Kitching et al. 2013). For large (in units of the PSF), bright objects such as those used here, this should be less of a problem. Moreover, after Gaussianization the PSF ellipticity is negligible. In this work we measure the shapes of member galaxies using two different methods, which allows us to test for consistency and robustness of the results. Below we give a brief outline of each method to highlight their differences; more details can be found in the original works.

Shapes are measured from the Gaussianized images (Sect. 2.1). The PSF in these images is, by construction, circular, Gaussian, and constant across the image. Therefore the shape measurement methods need to account for the blurring of the ellipticity by the PSF, but there are no systematic ellipticities in the images (to a high enough precision, see Sect. 4.3).

\subsubsection{Kaiser-Squires-Broadhurst (KSB)}

KSB was developed for weak lensing measurements by Kaiser et al. (1995) and revised by Hoekstra et al. (1998). It measures shapes by estimating the central second moments $I_{i j}$ of the image fluxes to measure the two-component polarization

$e_{1}=\frac{I_{11}-I_{22}}{I_{11}+I_{22}} ; \quad e_{2}=\frac{2 I_{12}}{I_{11}+I_{22}}$.

These measurents are weighted with a circular Gaussian of width $r_{\mathrm{g}}$, which corresponds to the radius of maximum significance measured by KSB; this weight reduces shot noise in the measurements. Blurring by the PSF is corrected by the so-called preseeing shear polarizability, $P^{\gamma}$, which quantifies the effect of the convolution of the PSF to the image polarization, $e_{i}$ (Luppino \& Kaiser 1997; Hoekstra et al. 1998). The corrected ellipticity is then $\epsilon_{i}=e_{i} / P^{\gamma 8}$. Both $e_{i}$ and $P^{\gamma}$ are measured with the same radius, $r_{\mathrm{g}}$, for each galaxy.

\subsubsection{GALFIT}

GALFIT was developed by Peng et al. (2002), having in mind the modeling of different components of galaxies for studies of galaxy structure and evolution. It attempts to model the light of a galaxy by fitting a multi-component generalized ellipse given by

$r=\left(|x|^{c+2}+\left|\frac{y}{q}\right|^{c+2}\right)^{1 /(c+2)}$,

where a true ellipse has $c=0$, a boxy shape $c>0$ and a disky shape $c<0$; here $q$ is the minor-to-major axes ratio. Additionally, the position angle, $\phi$, is defined as the direction of the major axis. GALFIT accounts for the PSF model (in this case a single Gaussian for each whole field) when measuring ellipticities. We use a simple Sérsic (1968) model for the surface brightness profile, $\ln I(r) \propto r^{1 / n}$. Only galaxies with Sérsic index $0.5<n<8$ and with axis ratio $q>0.15$ are included in the sample. We convert $q$ and $\phi$ to the same ellipticity measures of KSB through

$\epsilon_{1}=\left(\frac{1-q}{1+q}\right) \cos 2 \phi ; \quad \epsilon_{2}=\left(\frac{1-q}{1+q}\right) \sin 2 \phi$.

\footnotetext{
8 Because the PSF in our images has vanishing ellipticity by construction, the PSF correction of KSB is mathematically exact. This is not the case if the PSF is significantly elliptical.
}
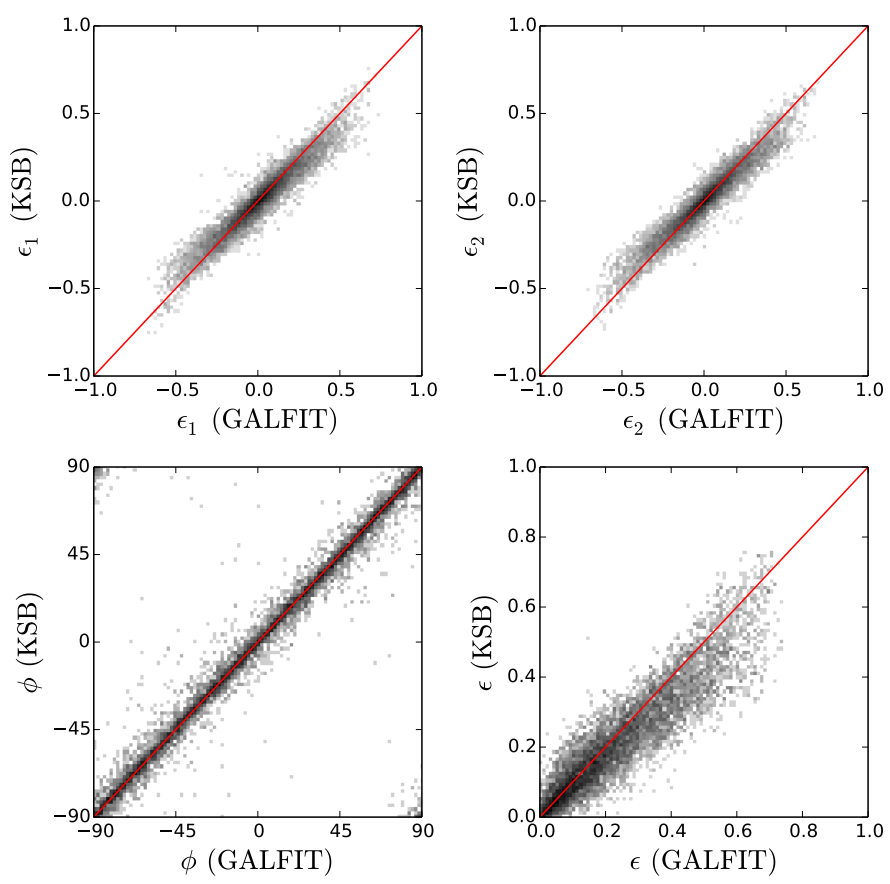

Fig. 6. Comparison of shape measurements from KSB and GALFIT. Grey scales show the number of points per bin in logarithmic scale. Red lines show $y=x$. Top: ellipticity components in Cartesian coordinates. Bottom left: position angles, $\phi$, in degrees. The periodicity of $\phi$ (of $180 \mathrm{deg}$ ) can be seen in the top left and bottom right corners of the plot. Bottom right: galaxy ellipticities.

\subsection{Systematic effects}

Because weak lensing measurements rely on averages of a large number of small signals, they are more prone to systematic effects than photometry and require more agressive masks. Therefore some spectroscopic members (all of which are in our photometric catalog) are not included in the shape catalogs. Moreover, the KSB and GALFIT catalogs are not the same since both have different requirements on, e.g., the size of an object and blending with nearby objects to estimate a reliable shape. Of the 14576 spectroscopic members, 13966 have a KSB shape measurement and 13360 have a GALFIT measurement, with an overlap of 12160 galaxies and a total of 14250 galaxies with a shape measurement. Similarly, of 23041 spectroscopic+RS members, 20493 have KSB measurements and 18511 have GALFIT measurements. The smaller number of objects with GALFIT measurements comes mainly from high-redshift galaxies (compare Tables 3 and 4). This is because small, faint galaxies are harder for GALFIT to fit, while KSB is well-suited to measure the shapes of faint (background) galaxies.

We only consider galaxies with shape measurements from either method in this work, except for the assessment of the red sequence in Sect. 3.2. Figure 6 compares the shape parameters for all spectroscopic members that have valid KSB and GALFIT estimates. While the measurements generally agree, there is a small but noticeable difference for large-ellipticity objects, such that KSB estimates lower ellipticities than GALFIT. This effect is present with more or less the same magnitude for all clusters; it is a genuine difference between the two methods (for our particular dataset), and there is an indication that this effect may be more pronounced for smaller objects. This difference is due to higher-order corrections that are not implemented in KSB, which become important at large ellipticities (Viola et al. 2011). 
Table 3. Average ellipticity components of spectroscopic members.

\begin{tabular}{l|rrrr|rrrr}
\hline \hline & \multicolumn{4}{|c|}{ KSB } & \multicolumn{4}{c}{ GALFIT } \\
\hline Sample & $N_{\text {gal }}{ }^{2}$ & \multicolumn{1}{|c}{$\left\langle\epsilon_{+}\right\rangle$} & \multicolumn{1}{c}{$\left\langle\epsilon_{\times}\right\rangle$} & $\sigma\left(\epsilon_{i}\right)^{b}$ & $N_{\text {gal }}{ }^{a}$ & \multicolumn{1}{c}{$\left\langle\epsilon_{+}\right\rangle$} & \multicolumn{1}{c}{$\left\langle\epsilon_{\times}\right\rangle$} & $\sigma\left(\epsilon_{i}\right)^{b}$ \\
\hline All & 8510 & -0.0037 & -0.0014 & 0.0027 & 8014 & 0.0004 & -0.0009 & 0.0031 \\
$z<0.14$ & 4170 & 0.0002 & 0.0000 & 0.0039 & 4612 & 0.0029 & -0.0003 & 0.0038 \\
$z \geq 0.14$ & 4340 & -0.0074 & -0.0027 & 0.0038 & 3402 & -0.0042 & -0.0020 & 0.0053 \\
$M_{200}<7 \times 10^{14} M_{\odot}$ & 2287 & -0.0059 & 0.0051 & 0.0052 & 2277 & -0.0057 & 0.0041 & 0.0057 \\
$M_{200} \geq 7 \times 10^{14} M_{\odot}$ & 6223 & -0.0029 & -0.0038 & 0.0032 & 5737 & 0.0030 & -0.0030 & 0.0037 \\
Relaxed & 3233 & -0.0037 & -0.0022 & 0.0044 & 3058 & -0.0038 & -0.0025 & 0.0050 \\
Disturbed & 5277 & -0.0036 & -0.0009 & 0.0034 & 4956 & 0.0031 & 0.0001 & 0.0040 \\
$M_{r} \leq-21$ & 4101 & -0.0031 & -0.0016 & 0.0039 & 3922 & 0.0009 & 0.0000 & 0.0044 \\
$M_{r}>-21$ & 4409 & -0.0042 & -0.0012 & 0.0038 & 4092 & -0.0001 & -0.0018 & 0.0044 \\
RS & 5806 & -0.0008 & -0.0009 & 0.0033 & 5595 & 0.0010 & -0.0001 & 0.0037 \\
Non-RS & 2704 & -0.0099 & -0.0025 & 0.0048 & 2419 & -0.0012 & -0.0031 & 0.0059 \\
\hline
\end{tabular}

Notes. ${ }^{(a)}$ Number of galaxies used for the average, within $r_{200} \cdot{ }^{(b)} 68 \%$ confidence measurement uncertainties on the average ellipticities.

Table 4. Average ellipticity components of spectroscopic plus red sequence members.

\begin{tabular}{l|rcrc|rrrr}
\hline \hline & \multicolumn{4}{|c|}{ KSB } & \multicolumn{4}{c}{ GALFIT } \\
\hline Sample & $N_{\text {gal }}$ & \multicolumn{1}{|c}{$\left\langle\epsilon_{+}\right\rangle$} & \multicolumn{1}{c}{$\left\langle\epsilon_{\times}\right\rangle$} & $\sigma\left(\epsilon_{i}\right)$ & $N_{\text {gal }}$ & \multicolumn{1}{c}{$\left\langle\epsilon_{+}\right\rangle$} & \multicolumn{1}{c}{$\left\langle\epsilon_{\times}\right\rangle$} & $\sigma\left(\epsilon_{i}\right)$ \\
\hline All & 15905 & -0.0022 & -0.0021 & 0.0020 & 12930 & 0.0000 & -0.0008 & 0.0026 \\
$z<0.14$ & 6407 & -0.0020 & -0.0019 & 0.0031 & 6233 & 0.0013 & 0.0002 & 0.0033 \\
$z \geq 0.14$ & 9498 & -0.0023 & -0.0022 & 0.0026 & 6697 & -0.0019 & -0.0023 & 0.0041 \\
$M_{200}<7 \times 10^{14} M_{\odot}$ & 5394 & -0.0039 & -0.0010 & 0.0034 & 4345 & -0.0057 & 0.0009 & 0.0044 \\
$M_{200} \geq 7 \times 10^{14} M_{\odot}$ & 10511 & -0.0013 & -0.0027 & 0.0024 & 8585 & 0.0031 & -0.0017 & 0.0032 \\
Relaxed & 7504 & -0.0034 & -0.0051 & 0.0029 & 5903 & -0.0044 & -0.0038 & 0.0038 \\
Disturbed & 8401 & -0.0011 & 0.0006 & 0.0027 & 7027 & 0.0039 & 0.0018 & 0.0035 \\
$M_{r} \leq-21$ & 5394 & -0.0020 & -0.0011 & 0.0034 & 4912 & 0.0008 & 0.0004 & 0.0040 \\
$M_{r}>-21$ & 10511 & -0.0023 & -0.0026 & 0.0024 & 8018 & -0.0005 & -0.0017 & 0.0034 \\
\hline
\end{tabular}

As we show in Sect. 5 this has no impact on our results, so we do not explore this issue further.

As a further test, Fig. 7 shows the alignment signals of the control samples. As expected, foreground galaxies have a signal consistent with zero in both ellipticity components at all radii, with large errorbars due to small statistics. The average ellipticities of stars are different from zero at significant levels in most of the radial range. However, the average ellipticity is constrained to $\left\langle\epsilon_{i}\right\rangle \lesssim 2 \times 10^{-4}$ at all radii, an order of magnitude smaller than the statistical errors in the alignments of cluster members. Thus any systematic effects arising from PSF uncertainty or other instrumental biases are controlled to much lower values than the statistical uncertainties, and can be neglected for the purposes of this work.

Finally, the Gaussianization of the images makes the PSF round and homogeneous across an image but produces anisotropic (correlated) noise, which could introduce noise bias in our measurements. The level of anisotropy can be assessed by measuring star ellipticities as a function of magnitude: if noise is highly anisotropic then noisier measurements would show, on average, a larger anisotropy than high- $\mathrm{S} / \mathrm{N}$ measurements. We test this by comparing the ellipticities of stars as a function of magnitude (for $18 \leq m_{r} \leq 22$ ), and find that the average ellipticities are consistent with the levels shown in Fig. 7. Moreover, we use galaxies whose number density drops rapidly beyond $m_{r} \sim 18$, and are typically 8 times larger than the PSF. We conclude that anisotropic noise can be safely neglected in this study.

\section{Results}

In this section we present and discuss the main results of this paper. We refer to Sect. 4 for details on the calculations that lead to the values reported here and a discussion of systematic effects.

\subsection{Satellite radial alignment}

Figure 8 shows the average radial alignment for all spectroscopically confirmed cluster members with good ellipticity measurements from KSB and GALFIT in annuli around the cluster center. Both methods show that the intrinsic alignment signal of cluster members is consistent with zero across all radii. Hereafter, we choose to quote average values within $r_{200}$ since, strictly speaking, this is the input required by the halo model (see Sect. 6). Within $r_{200}$, the alignment of spectroscopic members is constrained to an average of $\left\langle\epsilon_{+}\right\rangle=-0.0037 \pm 0.0027$ with KSB and $\left\langle\epsilon_{+}\right\rangle=0.0004 \pm 0.0031$ with GALFIT at $68 \%$ confidence. The cross components are also consistent with zero. Including red sequence members roughly doubles the number of galaxies used and confirms the latter result, with $\left\langle\epsilon_{+}\right\rangle=-0.0022 \pm 0.0020$ and $\left\langle\epsilon_{+}\right\rangle=0.0000 \pm 0.0026$ with KSB and GALFIT, respectively. 

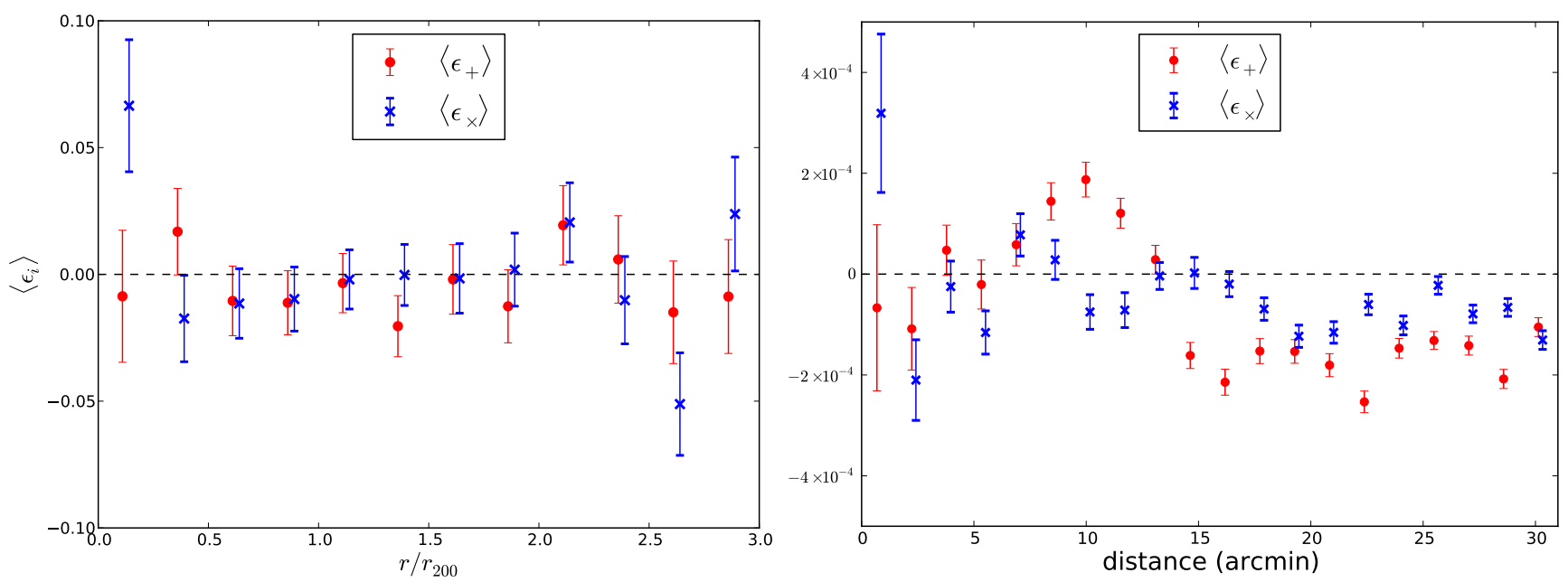

Fig. 7. Alignment signal from control samples measured with KSB, with data points shifted horizontally for clarity. Left: 3666 foreground galaxies in the direction of 73 clusters as a function of distance from the cluster, in units of $r_{200}$. Right: stars in the magnitude range $17<m_{r}<22$ as a function of angular distance from the center of each cluster. Typically, $r_{200} \sim 10^{\prime}$. Note the different vertical scales in each plot.

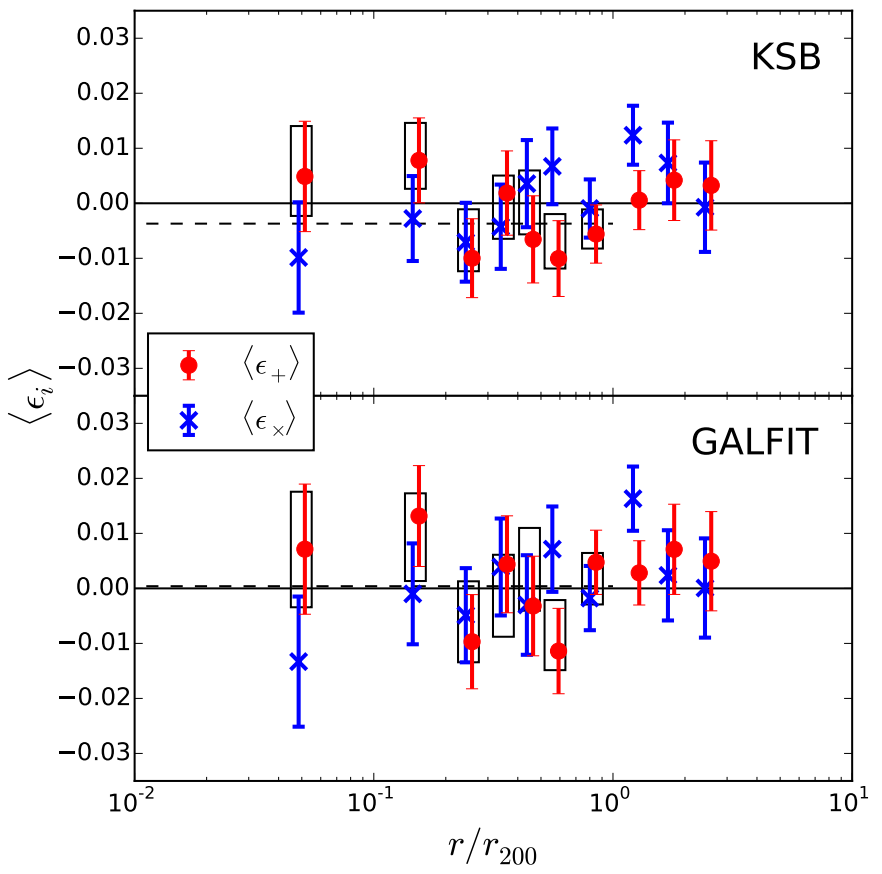

Fig. 8. Average alignment of all spectroscopically confirmed members out to $3 r_{200}$. The top panel shows the results from KSB while the bottom panel shows those from GALFIT. Shaded bands show the 1,2 and $3 \sigma$ uncertainties in the overall average and white bars show the $1 \sigma$ range for $\left\langle\epsilon_{+}\right\rangle$from the enhanced sample including red sequence members. Points are slightly shifted horizontally for clarity.

Our results are consistent with the nondetection of satellite radial alignments in massive clusters at $z>0.5$ (Hung \& Ebeling 2012), based on 500 spectroscopic members in the inner $\sim 500 \mathrm{kpc}$ of clusters, using imaging from the Hubble Space Telescope (HST), and also with measurements at smaller masses from photometrically-selected galaxy groups from SDSS (Hao et al. 2011; Chisari et al. 2014) and spectroscopically-selected galaxy groups from the Galaxy And Mass Assembly (GAMA) survey (Schneider et al. 2013). Our results suggest that the stars in galaxies within clusters do not feel a strong enough tidal torque to be aligned toward the center of the cluster, in contrast with results from simulations which find strong alignments even when accounting for differences in the response between stars and dark matter which naturally occurs in hydrodynamical simulations (Pereira \& Bryan 2010; Tenneti et al. 2014; Velliscig et al., in prep.). An obvious consideration from the observational point of view is miscentering: whether the chosen cluster center is really the minimum of the cluster potential. This effect can be measured statistically with stacked weak lensing measurements (e.g., George et al. 2012) but is otherwise hard to assess observationally. At least in very relaxed clusters, BCGs are typically very close to the peak of the gas distribution (e.g., Lin \& Mohr 2004; Mahdavi et al. 2013), which is closely matched to the dark matter distribution (Faltenbacher et al. 2007b). We can therefore test, to some extent, whether miscentering could be diluting an alignment signal by isolating relaxed clusters as discussed in Sect. 3.1.1. However, as shown in the top panel of Fig. 9, we do not detect any alignment signal neither from relaxed nor from disturbed clusters. Thus we conclude that our results are robust to miscentering effects and that, statistically, satellite galaxies do not align toward the centers of clusters.

As discussed by Hao et al. (2011), the redshift evolution of satellite radial alignments, if any, contains valuable information as to whether these alignments are produced during the formation of clusters or an evolving product of tidal torques within clusters. The middle panel of Fig. 9 shows that the alignment signal is consistent with zero across redshift, suggesting that neither of these processes is sufficient to sustain radial alignments over cosmological time. Furthermore, the bottom panel of Fig. 9 shows that this nondetection is also independent of cluster mass. We further tested whether any orientation bias, in the sense that we might have clusters viewed preferentially along their major axis, could have any effects on our results. To do this, we divided the cluster sample by BCG elongation, assuming that BCGs that look rounder might actually be elongated along the line-of-sight. Both cluster samples have radial alignments consistent with zero (not shown), arguing that a possible orientation bias is not a problem here.

In any of the two scenarios mentioned above (namely tidal and primordial alignments), radial alignments could show a different pattern for galaxies with different formation histories. We investigate this by splitting the galaxy sample by galaxy luminosity (as a proxy for galaxy mass) and color - since bluer galaxies have been accreted more recently. To split by galaxy color we 


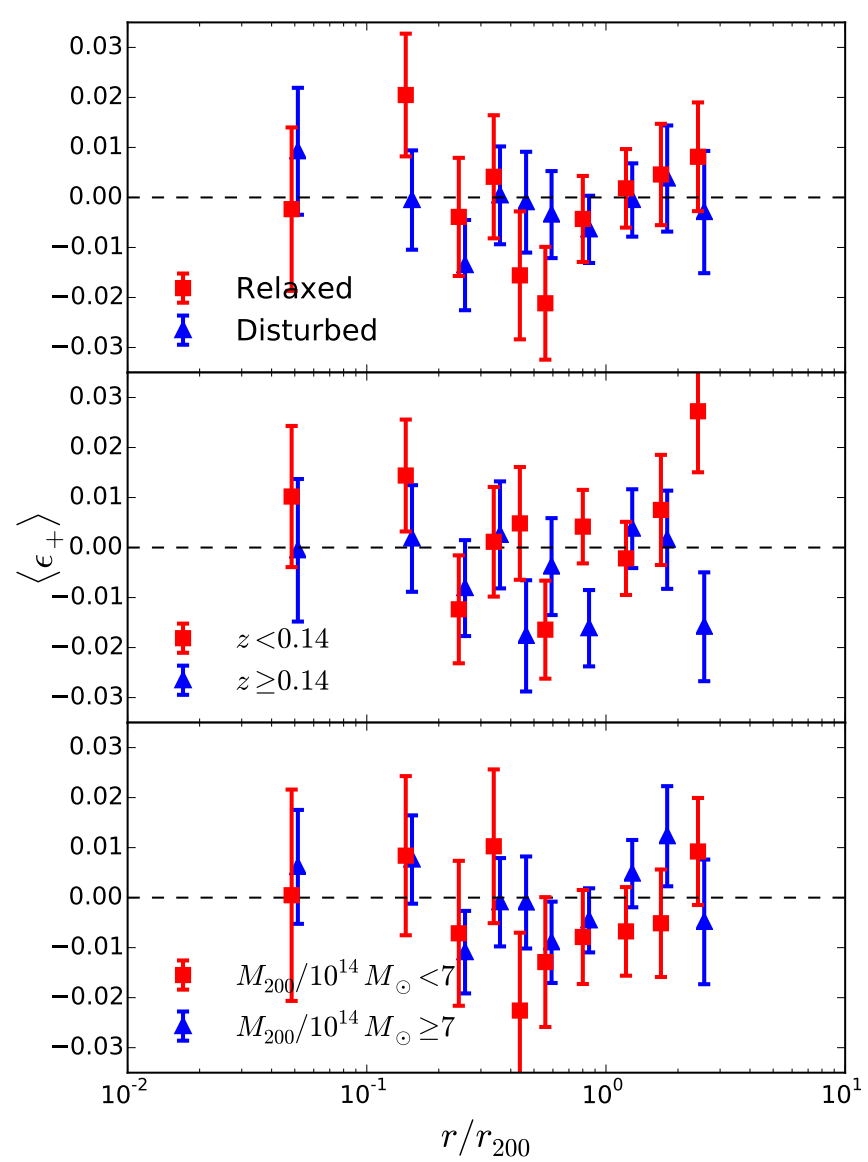

Fig. 9. Average alignment $\left\langle\epsilon_{+}\right\rangle$from KSB for spectroscopically confirmed members, divided by cluster properties: by dynamical state (top; see Sect. 3.1.1), redshift (middle), and dynamical mass (bottom). The latter two thresholds are the median values of the cluster sample.

use each cluster's red sequence, which depends linearly on apparent magnitude, as outlined in Sect. 3.2. As seen in Fig. 10, we find no radial alignments consistently across galaxy colors and luminosities.

The results discussed above are summarized in Tables 3 and 4 for spectroscopic and spectroscopic plus red sequence member samples, respectively.

\subsection{Satellite-BCG alignment}

The second type of alignment we explore is the alignment of the satellite orientations with the BCG orientation (cf. Eq. (7)). A large number of observations suggest that BCGs are on average oriented along the major axes of clusters themselves (e.g., Sastry 1968; Binggeli 1982; Faltenbacher et al. 2007; Niederste-Ostholt et al. 2010; Hao et al. 2011), and there is evidence that the velocity dispersion of satellite galaxies is typically larger along the BCG major axis (Skielboe et al. 2012). It is possible, then, that the BCG orientation represents a preferred infall direction. If this is the case, it is possible that galaxies would be aligned toward this infall direction.

Figure 11 shows the alignment of galaxies with the major axis of the BCGs measured with $\mathrm{KSB}$ as a function of radius, for the full sample of spectroscopic plus red sequence members. As in the case of radial alignments, the data are also consistent with no satellite-BCG alignments at all distances. The average KSB signal within $r_{200}$ is $\left\langle\epsilon_{1}^{\prime}\right\rangle=-0.0021 \pm 0.0022$; the average

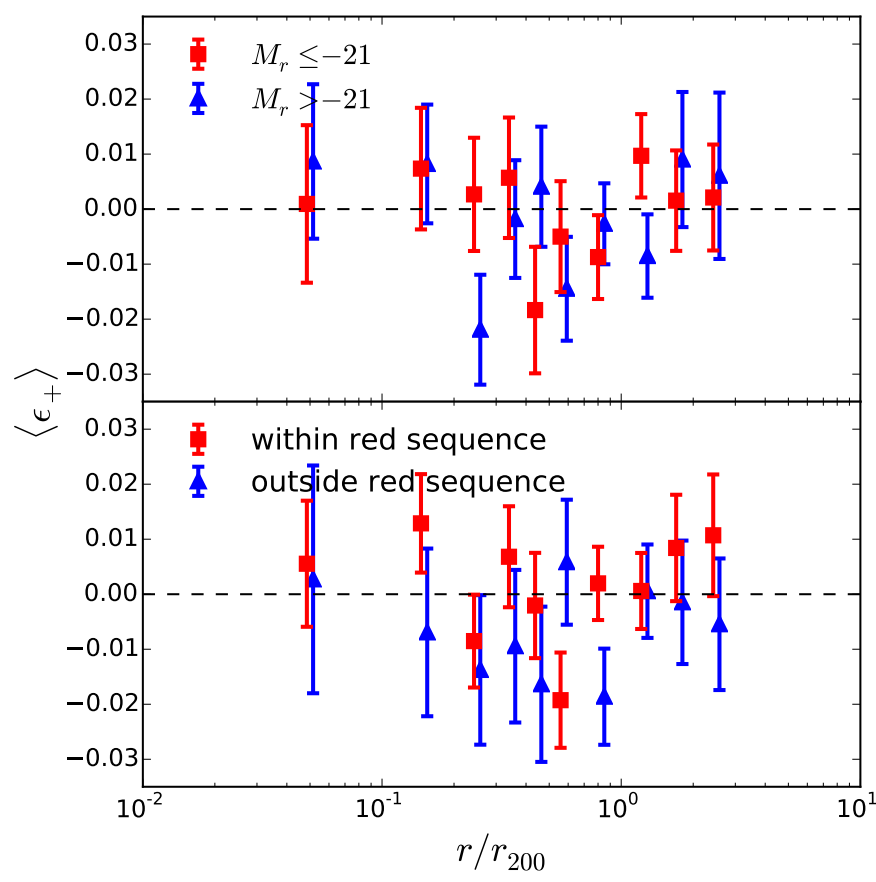

Fig. 10. Average alignment $\left\langle\epsilon_{+}\right\rangle$from KSB for spectroscopically confirmed members divided by galaxy properties: by rest-frame $r$-band absolute magnitude (top), and color with respect to each cluster's red sequence (bottom)

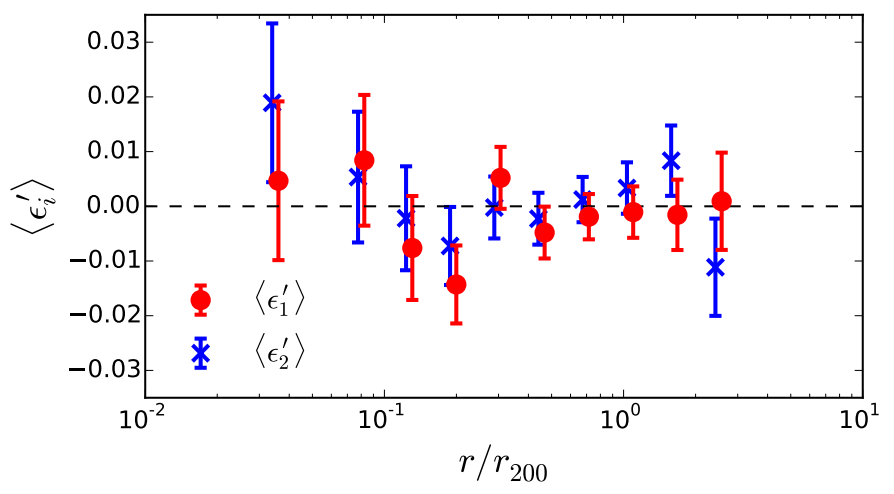

Fig. 11. Mean ellipticity components of spectroscopic plus red sequence satellite galaxies in a frame rotated by the position angle of the BCG, probing the alignment of satellites with the cluster BCG. BCG position angles are measured with GALFIT, while the shapes of satellite galaxies are measured with KSB. Red circles show alignments with respect to the major $\left(\epsilon_{1}^{\prime}>0\right)$ and minor $\left(\epsilon_{1}^{\prime}<0\right)$ axes of the BCG, while blue crosses show alignments at $45^{\circ}$ rotations.

GALFIT signal is $\left\langle\epsilon_{1}^{\prime}\right\rangle=-0.0024 \pm 0.0029$. We also split the sample as in the preceeding section, and find no signal for all galaxy and cluster subsamples. As a consistency check, we also find that the distribution of position angles, $\left|\phi-\phi_{\mathrm{BCG}}\right|$, is consistent with a random distribution.

Finally, we averaged not in annular bins but in Cartesian coordinates $\{x, y\}$, to check if the satellite-BCG alignment could be happening only along a preferential direction, such that the azimuthal average would dilute the signal. We also found a null signal in this case (not shown).

\subsection{Satellite-satellite alignment}

We have shown in Sect. 5.1 that satellite galaxies are not aligned toward the centers of clusters. If galaxies reside within 


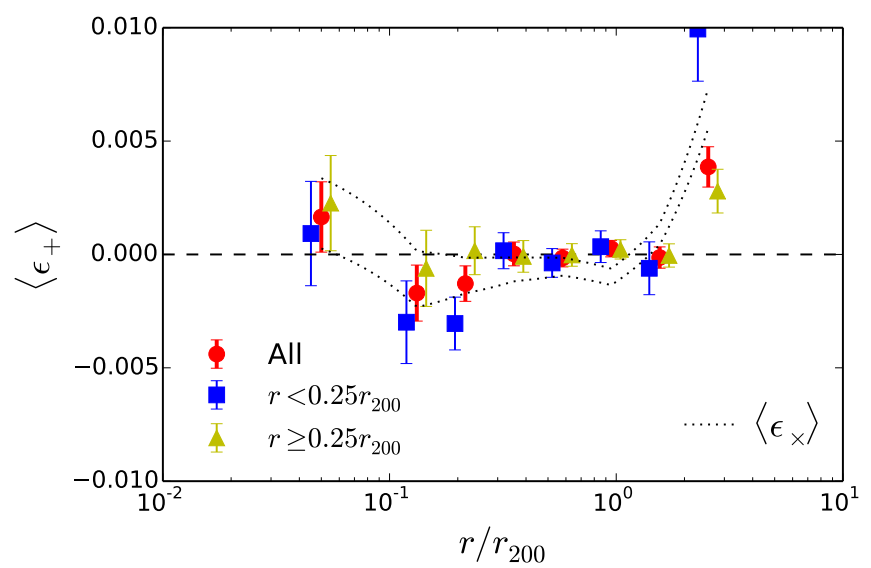

Fig. 12. Satellite-satellite alignment as a function of the distance between satellites, for the full spectroscopic plus red sequence member sample. Red circles show all galaxies, while blue squares and yellow triangles show the signal with respect to galaxies inside and outside $0.25 r_{200}$. Data points show the radial (positive) and tangential (negative) signal, while the dashed lines show the $68 \%$ range of the cross component, linearly interpolated. Uncertainties do not account for covariance between data points. Note that the vertical scale is smaller than in Figs. 8-11.

substructures themselves, then these substructures might have tidally aligned galaxies toward them. If the tidal torque of the cluster is not enough to overcome these substructure-scale alignments, then maybe we can observe an alignment signal at small separations, between satellite galaxies. After excluding data near the edges of the images (see Sect. 4.1.3), we use a total of $3.93 \times 10^{6}$ satellite pairs. Figure 12 shows the alignment signal between satellites averaged over all clusters, as a function of distance between satellites, for the full spectroscopic plus red sequence member sample. In this case we split the sample into two radial bins, namely (test) galaxies within and outside $0.25 r_{200}$, which corresponds to the scale radius of a cluster with a concentration $c_{200}=4$ (roughly what is expected for massive clusters; e.g., Duffy et al. 2008), but the results are similar when splitting the sample at other radii.

The leftmost bins in Fig. 12 show the signal from substructure: outer bins probe the radial alignment between galaxies at large distances. It might be expected that substructure in the outskirts of clusters would contain an alignment signal since, presumably, they have been accreted more recently. As in the preceding sections, we do not observe any alignment signal for the full cluster sample, nor for relaxed or disturbed clusters, at any radii. We note however that the last data point in Fig. 12 is significantly nonzero, but so is the cross component. This suggests that at these distances measurements are affected by systematic effects, mainly because a large fraction of the pairs consist of two galaxies at the edges of the images. Moreover, this data point shows the alignments between satellites at opposite sides of the cluster; i.e., it is not a measurement of alignment within cluster substructure.

Since we do not detect any alignment signal for clusters at different redshifts and at different dynamical stages, we conclude that tidal torques in clusters, or in substructures within them, do not result in significant alignments of the stellar content of galaxies at any scale (neither toward the center nor between galaxies). It may be possible to bring this in line with the strong alignments measured in $N$-body simulations by invoking a misalignment between the stellar and dark matter distributions (e.g., Okumura et al. 2009; Tenneti et al. 2014). However, such analysis is beyond the scope of this paper.

\subsection{Is there an agreement on the level of galaxy alignments in groups and clusters?}

As discussed above, previous studies have reported various levels of alignment of satellite galaxies in clusters using different estimators. We expect such a lack of agreement to arise for two main reasons: the quality of the images used to measure galaxy shape parameters, and the use of shape measurements that are prone to systematic effects, e.g., isophotal measurements. The latter effect was studied by Hao et al. (2011) in detail; they found significant radial alignments only when using isophotal shape measurements, and that the strength of these alignments depends on apparent magnitude but not on absolute magnitude, a strong suggestion that the detection is an artifact. Specifically, isophotal measurements are subject to severe contamination from the $\mathrm{BCG}$, which can extend over a few hundred kpc in the case of massive clusters. As to the first cause, the quality of imaging data used by different groups varies significantly. To our knowledge, Plionis et al. (2003) were the first to use CCD photometry to measure galaxy alignments. They found a significant anisotropy in the (isophotal) position angles of satellite galaxies of Abell 521 (though they used photographic plates for their statistical study of alignments in clusters). There are also recent studies, however, who used position angle measurements extracted from scanned photographic plates (e.g., Baier et al. 2003; Panko et al. 2009; Godłowski et al. 2010), both of which are of noticeably lower quality than present-day observations. Moreover, these works typically used single-band information to select cluster members, yielding an unknown (and likely low) sample purity.

Most recent studies have used data from SDSS because of its unmatched statistical power. These data, while of very high quality compared to photographic plate measurements, are several magnitudes shallower than our MegaCam data and taken under less ideal conditions (with seeing a factor 2 larger). Conversely, Hung \& Ebeling (2012) have used deep, high-quality HST imaging to measure galaxy alignments, finding no evidence for galaxy alignments within clusters. As in our analysis, Hung \& Ebeling (2012) have considered spectroscopicallyconfirmed cluster members, thus in addition to the superior photometry, both works have a cleaner member sample, which is key to the interpretation of the signal. Schneider et al. (2013) also used a sample of spectroscopically-confirmed group members, plus a shape measurement method that was specifically calibrated to weak lensing measurements (Mandelbaum et al. 2005), and found no significant evidence for alignments. Finally, Chisari et al. (2014) measured galaxy alignments in photometrically-selected galaxy groups and clusters in SDSS Stripe 82, fully accounting for photometric redshift uncertainties, and constrain alignments to similar values as those found here.

The fact that all recent measurements that use high-quality imaging and properly calibrated shape measurements have yielded null detections (Hao et al. 2011; Hung \& Ebeling 2012; Schneider et al. 2013; Chisari et al. 2014, plus the present study) leads us to conclude that there is no evidence for intrinsic alignments of satellite galaxies in galaxy groups or clusters to the level of uncertainty achievable with current datasets (both statistical and systematic). 


\section{Contamination to cosmic shear measurements}

In this section we explore the impact that the measured galaxy alignments in clusters can have on future cosmic shear measurements. We quantify the contribution of intrinsic alignments to cosmic shear measurements through the matter and intrinsic alignment power spectra, which can be defined as

$$
\begin{aligned}
\left\langle\tilde{\gamma}^{I *}(\boldsymbol{k}) \tilde{\gamma}^{I}\left(\boldsymbol{k}^{\prime}\right)\right\rangle & =(2 \pi)^{3} \delta_{D}^{(3)}\left(\boldsymbol{k}-\boldsymbol{k}^{\prime}\right) P_{\mathrm{II}}(\boldsymbol{k}) \\
\left\langle\delta^{*}(\boldsymbol{k}) \tilde{\gamma}^{I}\left(\boldsymbol{k}^{\prime}\right)\right\rangle & =(2 \pi)^{3} \delta_{D}^{(3)}\left(\boldsymbol{k}-\boldsymbol{k}^{\prime}\right) P_{\mathrm{GI}}(\boldsymbol{k}) .
\end{aligned}
$$

Here, $\tilde{\gamma}^{I}=\left(1+\delta_{\mathrm{g}}\right) \gamma^{I}$ is the (projected) ellipticity field weighted by the galaxy density, $\delta_{\mathrm{g}}$, and $P_{\mathrm{II}}(\boldsymbol{k})$ and $P_{\mathrm{GI}}(\boldsymbol{k})$ are the II and GI contributions to the power spectrum including a prescription for nonlinear evolution (i.e., nonlinear power spectra, see Smith et al. 2003; Bridle \& King 2007), respectively; $\delta^{*}$ is the complex conjugate of the Fourier transform of the matter density contrast, $\delta(\boldsymbol{r})=(\rho(\boldsymbol{r})-\bar{\rho}) / \bar{\rho}$ is the matter overdensity with respect to the average density of the Universe, $\tilde{\gamma}^{I *}$ indicates the complex conjugate of $\tilde{\gamma}^{I}$, and $\delta_{D}$ is a Dirac delta function.

Additionally, we translate the 3-dimensional power spectra discussed above into (observable) angular power spectra, $C_{\ell}$, using the Limber (1953) approximation (e.g., Kaiser 1992). We use a source redshift distribution given by

$p(z) \propto z^{\alpha} \exp \left[-\left(z / z_{0}\right)^{\beta}\right]$,

where we fix the parameters $\alpha, \beta$, and $z_{0}$ so that the median redshift of the model distribution reproduces the median redshift of the Kilo-Degree Survey (KiDS; de Jong et al. 2013), $z_{\text {med }} \simeq 0.7$ (Kuijken et al., in prep.). We split the lens sample in redshift bins of half-width $\Delta z=0.1$ to illustrate the results obtained from a tomographic cosmic shear analysis (e.g., Heymans et al. 2013). We use a narrow redshift bin covering $0.6<z<0.8$ for the GG and II power spectra, since this range is close to the one that maximizes the lensing signal in a KiDS-like tomographic analysis. The GI power spectrum is better captured by crosscorrelating this redshift bin with one at low redshift; we choose $0.2<z<0.4$ as a compromise between a high intrinsic alignment efficiency and a large enough volume observed.

\subsection{Linear alignment model}

The simplest models for galaxy alignments predict that elliptical galaxies are aligned with a strength that is proportional to the tidal field (Catelan et al. 2001) while spiral galaxies, which are aligned by angular momentum acquired during gravitational collapse, are aligned with a strength that is proportional to the square of the tidal field (Pen et al. 2000). On sufficiently large scales, all galaxies are predicted to experience an alignment proportional to the large scale gravitational potential (Hui \& Zhang 2002). Thus a linear alignment model is usually employed to characterize large scale galaxy alignments (e.g., Kirk et al. 2010; Joachimi et al. 2011; Mandelbaum et al. 2011; Heymans et al. $2013)^{9}$. We normalize the intrinsic alignment power spectra as in previous studies (Hirata \& Seljak 2004; Bridle \& King 2007; Schneider \& Bridle 2010), matching to the SuperCOSMOS measurements of Brown et al. (2002). This normalization is also

\footnotetext{
9 This model is typically referred to as "nonlinear alignment model". However, this is a misnomer, since intrinsic alignments are still modeled as depending linearly on the tidal field; instead the name arises from the use of the nonlinear power spectrum in Eq. (11). We therefore refer to it as linear alignment model throughout.
}

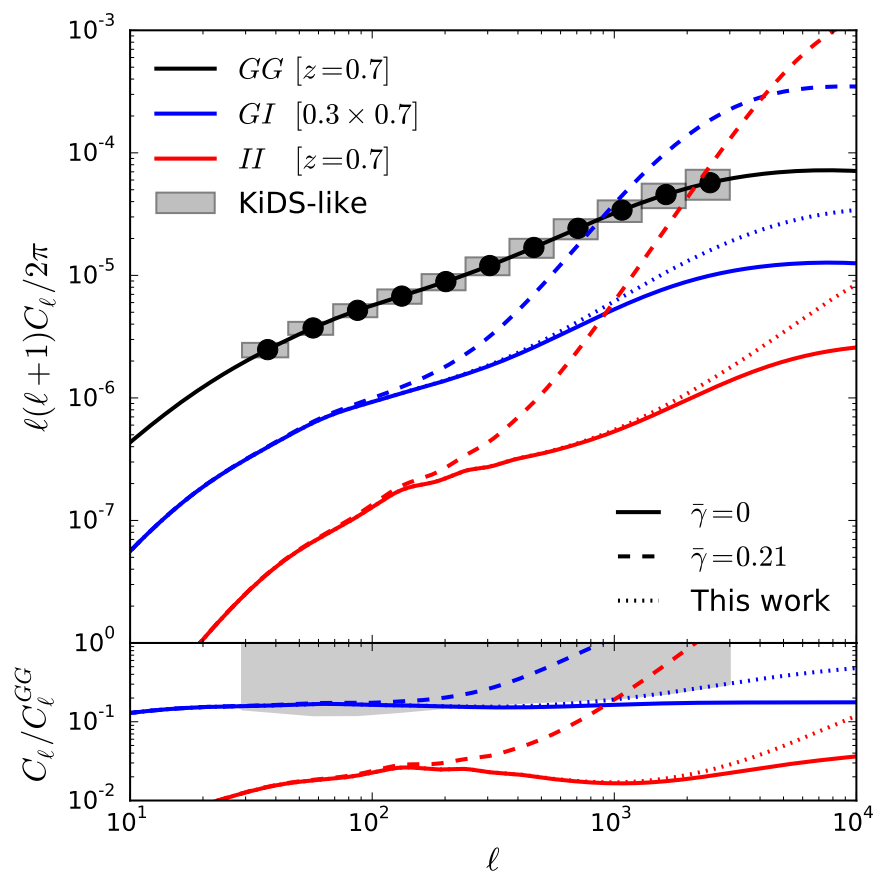

Fig. 13. Effect of intrinsic alignments on the angular power spectra. Top panel: The black line shows the GG power spectrum, while blue and red lines show the GI and II power spectra, respectively. Solid lines show the linear alignment model with no small-scale intrinsic alignments, while dashed and dotted lines model the contribution of satellite galaxies with $\bar{\gamma}=0.21$ as in Schneider \& Bridle (2010) and with the mass-dependent $2 \sigma$ upper limit on the alignment signal derived in this work (see Sect. 6.3), respectively. Grey boxes with black circles show the expected uncertainty levels on a KiDS-like survey covering 1500 sq. deg and with $n_{\text {gal }}=10 \mathrm{arcmin}^{-2}$. Bottom panel: ratio between the GI and II power spectra and the GG power spectrum, for each model. The shaded region shows values above the $1 \sigma$ uncertainties in the anglar power spectrum for a KiDS-like survey, where GI and II contributions would dominate over statistical uncertainties.

consistent with more recent observations (Heymans et al. 2004; Mandelbaum et al. 2006; Joachimi et al. 2011).

Solid lines in Fig. 13 show the angular power spectra, $C_{\ell}$, from the linear alignment model. This model includes no contribution from alignments within halos (so-called 1-halo terms) and therefore the II and GI power spectra are subdominant to the matter power spectrum at all scales.

Figure 13 also shows the expected angular power spectrum measurements of a reference cosmic shear survey with properties similar to KiDS with a redshift distribution as described above, with a sky coverage of 1500 sq. deg and a background source density of $n_{\text {gal }}=10 \mathrm{arcmin}^{-2}$. We assume a coverage $30 \leq \ell \leq 3000$, and compute the expected $C_{\ell}$ measurements and uncertainties following Cooray \& $\mathrm{Hu}$ (2001), in logarithmic bins in $\ell$. The bottom panel of Fig. 13 shows that the II contribution remains safely subdominant to statistical uncertainties expected for KiDS, but the GI contribution cannot be ignored, contaminating the GG power spectrum at the $\sim 10 \%$ level.

\subsection{Halo model}

The linear alignment model aims to describe alignments at large scales and the alignments between central galaxies, because these are expected to be aligned with the host halo by the large scale gravitational potential. On smaller, nonlinear scales, galaxy formation will tend to misalign baryonic and dark matter (e.g., 
Pereira \& Bryan 2010; Tenneti et al. 2014), so the large-scale results from $N$-body simulations are probably not directly applicable to galaxy alignments within halos. Galaxy formation can also have a major impact on the power spectra (van Daalen et al. 2011; Semboloni et al. 2011), and the way these two effects interplay is unclear. We therefore require a prescription to predict the power spectra accounting for 1-halo term galaxy alignments. To this end, we employ the halo model of radial alignments introduced by Schneider \& Bridle (2010).

The main assumption of the halo model is that galaxies form and reside in dark matter haloes whose masses directly influence the (observable) properties of the galaxies they host. Additionally, one can assume that satellite galaxies in a halo are radially aligned toward the center with a strength that can in principle be a function of the galaxy position in the halo, the host halo mass, and redshift. This is known as a satellite radial alignment model. The total alignment can be separated into a prescription for galaxies in halos (the 1-halo term), and one between halos (the 2-halo term). We assume that galaxies populate halos following the halo occupation distribution of Cacciato et al. (2013) and the halo mass and bias functions of Tinker et al. (2010). More details about the ingredients of this halo model can be found in Schneider \& Bridle (2010). Given a model for radial alignments, $\gamma^{I}(\mathbf{r}, M, z)$, we calculate the power spectra through Eq. (11).

This model only incorporates the information about the radial alignments studied in Sect. 5.1, by definition. In principle, it would be possible to include further constraints on the alignments from measurements such as those explored in Sects. 5.2 and 5.3. However, these would be second order corrections to the cluster-scale radial component. In particular, the satellite-satellite alignment constraints woud be relevant on scales smaller than what will be probed by current and upcoming experiments; we therefore choose not to include them in the present analysis.

\subsection{Impact of alignments within halos on the power spectra}

The halo model requires a prescription for the strength of smallscale radial alignments. In its simplest form this strength is constant with radius and halo mass. The power spectra derived from this model are shown as dashed lines in Fig. 13, for an alignment strength $\bar{\gamma}=0.21$ ( $\bar{\gamma}$ is the 3-dimensional alignment strength derived from a projected measurement, $\gamma^{I}$; see Schneider \& Bridle 2010). This is the fiducial value adopted by Schneider \& Bridle (2010). In this work, we extend this prescription by assuming a radial alignment that depends on halo mass but not on distance within the halo. Such a model is fully consistent with our results, since we find a null signal at all radii.

We construct a mass-dependent alignment model using the present results, plus the intrinsic alignment measurements in galaxy groups from the Galaxy and Mass Assembly (GAMA) survey (Schneider et al. 2013). We assume a power law for $\gamma^{I}(M)$, such that the mean ellipticity of satellite galaxies has the $2 \sigma$ upper limits obtained in this study. We use the results for the augmented spectroscopic plus red sequence member sample, and choose to use the KSB measurement because, although the GALFIT constraint is less tight (i.e., more conservative), the contribution of each mass scale is weighted by the mass function. Since the mass function is an exponential function of mass, the overall alignment signal is dominated by lower mass objects. Since we use the constraint found for GAMA groups as a pivot, a smaller alignment strength in clusters will mean a larger overall contribution of alignments to a cosmic shear survey. Specifically, we use $\epsilon_{+}<0.0019$ at $M \simeq 10^{15} M_{\odot}$ and $\epsilon_{+}<0.019$ at a typical mass $M \simeq 10^{13} M_{\odot}$, corresponding to the $2 \sigma$ upper limit for GAMA groups with $N_{\text {gal }} \geq 5$ (Schneider et al. 2013). Our model is therefore $\gamma^{I}(M)=\left(M / M_{0}\right)^{\alpha}$, constant with redshift, with $M_{0}=1.19 \times 10^{9} M_{\odot}$ and $\alpha=-0.5^{10}$. We note that the assumption of a single power law at all masses has no justification other than its simplicity. A more detailed halo model for intrinsic alignments will be presented in a forthcoming study (Cacciato et al., in prep.), where we explore the impact of halo model assumptions on the predictions of the II and GI power spectra.

Dotted lines in Fig. 13 show the intrinsic alignment power spectra predicted by the halo model for our adopted $\gamma^{I}(M)$. Since we constructed the model using $2 \sigma$ upper limits on the measured alignments, the regions between the solid and dotted lines should be regarded as conservative estimates of the current uncertainties on the GI and II power spectra due to 1-halo term intrinsic alignments. As can be seen, both the GI and II power spectra remain subdominant to the GG power spectrum, which is not the case with the fiducial $\bar{\gamma}=0.21$ model used by Schneider \& Bridle (2010). The GI angular power spectrum including our 1-halo term is $\sim 70 \%$ higher than that predicted by the linear alignment model at $\ell \sim 3000$, which translates into an excess on the total $(\mathrm{GG}+\mathrm{GI}+\mathrm{II})$ angular power spectrum of $\approx 10 \%$, comparable to the statistical uncertainties expected at these scales. Note that at larger scales the GI power spectrum is dominated by linear alignments and the satellite contribution is well below the statistical uncertainties of KiDS. Therefore, we do not expect that cosmic shear analyses with KiDS will need to include a contribution by satellite galaxies in the modeling of intrinsic alignments, and we conclude that the linear alignment model should be a sufficient treatment of intrinsic alignments for KiDS. We note that for a bin at $0.2<z<0.4$, the II power spectrum can be $>10 \%$ of the lensing (GG) power spectrum at the same redshift, but the uncertainties of a KiDS-like survey are much larger than at $z \sim 0.8$ because of the smaller volume probed. In any case, the linear alignment model captures any II contribution to sufficient accuracy. Therefore a treatment of intrinsic alignments in KiDS cosmic shear analyses can rely on the linear alignment model, similar to the cosmic shear analysis of CFHTLenS data by Heymans et al. (2013). We expect the situation to be similar for the Dark Energy Survey (DES $)^{11}$, which will have three times as much area as KiDS but otherwise similar characteristics. This may not be the case for larger surveys, for which the contribution of satellite galaxies to intrinsic alignments must be characterized to higher precision.

\section{Conclusions}

We have compiled a large sample of galaxies with spectroscopic redshifts in the direction of 90 galaxy clusters in the redshift range $0.05<z<0.55$, selected as part of MENeaCS and CCCP. We select cluster members using the shifting gapper technique, which uses phase space information, for a total 14576 cluster members. We use these members to estimate dynamical masses using the simulation-based scaling relation between velocity dispersion and cluster mass of Evrard et al. (2008). The sample has a median redshift $z=0.14$ and a median mass $M_{200} \sim 7 \times 10^{14} M_{\odot}$, in good agreement with the weak lensing masses estimated by Hoekstra et al. (2012).

\footnotetext{
10 The conversion between ellipticity and shear is given by $\gamma=\epsilon_{+} / 2 \mathcal{R}$, where $\mathcal{R}$ is the shear responsivity, which we assume to be equal to 0.87 . 11 http: //WwW . darkenergysurvey.org
} 
We quantify the alignment of galaxies within clusters using 14250 cluster members for which we are able to measure their shapes either with KSB or GALFIT, after showing that the ellipticities measured by both methods are consistent (Fig. 6). Both methods take different approaches to measuring galaxy shapes and therefore provide an important consistency check. We confirm that our analysis is free of significant systematic effects by measuring the average alignment of both foreground galaxies and stars. The signal from foreground galaxies is consistent with zero; the signal from stars is significantly different from zero, but at a level of $\left\langle\epsilon_{+}\right\rangle \sim 10^{-4}$, an order of magnitude lower than measurement uncertainties (Fig. 7).

We measure three different alignments: the radial alignment of satellite galaxies toward the BCG, the alignment of satellites with the BCG orientation, and the radial alignment of satellites toward each other. Each probes a different, but not necessarily independent, effect. We find no evidence for any of these alignments (Figs. 8-12). In particular, we constrain the average ellipticity of satellites toward BCGs to $\left\langle\epsilon_{+}\right\rangle=-0.0037 \pm 0.0027$ with KSB and $\left\langle\epsilon_{+}\right\rangle=0.0004 \pm 0.0031$ with GALFIT, at $68 \%$ confidence, within $r_{200}$. Similarly, there is no evidence of galaxy alignments when splitting the sample by cluster (redshift, mass, or dynamical state) or galaxy (color or luminosity) properties. Selecting additional cluster members through the red sequence allows us to extend the sample to 20000 galaxies with an estimated contamination of $<10 \%$ from red sequence interlopers (Fig. 4). All signals from this enlarged sample are also consistent with zero.

We include this constraint on the radial alignment of galaxies within high-mass halos, together with a measurement at the group scale (Schneider et al. 2013), in a halo model framework, and derive the current uncertainty on the angular power spectrum given by intrinsic alignments within halos (a 1-halo term). We find that the total (GG+GI+II) angular power spectrum predicted from our alignment model (see Sect. 6.3) is, at most, $10 \%$ higher than the total power spectrum predicted by the linear alignment model at the smallest scales probed by KiDS, $\ell \sim 3000$. This level of contribution from satellite galaxies will not be relevant for cosmic shear measurements with KiDS or DES (see Fig. 13). We conclude that the linear alignment model is a sufficient description of intrinsic alignments for KiDS, but the situation may be different for significantly larger surveys.

Acknowledgements. We thank Chris Pritchet and Dennis Zaritsky for help carrying out the MENeaCS spectroscopic survey, Patrick Kelly for making his python SLR code publicly available, and Radek Wojtak for suggesting an assessment of orientation bias. C.S., H.H., and M.V. acknowledge support from the European Research Council under FP7 grant number 279396. H.H., M.C and R.F.J.vdB. acknowledge support from the Netherlands Organisation for Scientific Research grant number 639.042.814. Based on observations obtained with MegaPrime/MegaCam, a joint project of CFHT and CEA/IRFU, at the CanadaFrance-Hawaii Telescope (CFHT) which is operated by the National Research Council (NRC) of Canada, the Institut National des Science de l'Univers of the Centre National de la Recherche Scientifique (CNRS) of France, and the University of Hawaii. Funding for SDSS-III has been provided by the Alfred P. Sloan Foundation, the Participating Institutions, the National Science Foundation, and the U.S. Department of Energy Office of Science. The SDSS-III web site is http: //wwW . sdss3.org/. This research has made use of the NASA/IPAC Extragalactic Database (NED) which is operated by the Jet Propulsion Laboratory, California Institute of Technology, under contract with the National Aeronautics and Space Administration. This publication makes use of data products from the Two Micron All Sky Survey, which is a joint project of the University of Massachusetts and the Infrared Processing and Analysis Center/California Institute of Technology, funded by the National Aeronautics and Space Administration and the National Science Foundation. This research has made use of NASA's Astrophysics Data System Bibliographic Services. This work has made use of the python packages scipy (http://www. scipy.org) and matplotlib (http://matplotlib.org; Hunter 2007) and of IPython (Pérez \& Granger 2007).

\section{References}

Abraham, R. G., Yee, H. K. C., Ellingson, E., Carlberg, R. G., \& Gravel, P. 1998, ApJS, 116, 231

Agustsson, I., \& Brainerd, T. G. 2006, ApJ, 644, L25

Ahn, C. P., Alexandroff, R., Allende Prieto, C., et al. 2014, ApJS, 211, 17

Altay, G., Colberg, J. M., \& Croft, R. A. C. 2006, MNRAS, 370, 1422

Baier, F. W., Godłowski, W., MacGillivray, H. T. 2003, A\&A, 403, 847

Barrena, R., Boschin, W., Girardi, M., \& Spolaor, M. 2007, A\&A, 469, 861

Barrena, R., Girardi, M., \& Boschin, W. 2011, A\&A, 529, A128

Barrena, R., Girardi, M., \& Boschin, W. 2013, MNRAS, 430, 3453

Beers, T. C., Flynn, K., \& Gebhardt, K. 1990, AJ, 100, 32

Belloni, P., \& Roser, H.-J. 1996, A\&AS, 118, 65

Bernstein, G. M., \& Norberg, P. 2002, AJ, 124, 733

Bertin, E., \& Arnouts, S. 1996, A\&AS, 317, 393

Binggeli, B. 1982, A\&A, 107, 752

Blazek, J., Mandelbaum, R., Seljak, U., \& Nakajima, R. 2012, J. Cosmol. Astropart. Phys., 05, 041

Boschin, W., Girardi, M., Barrena, A., et al. 2004, A\&A, 416, 839

Boschin, W., Barrena, R., \& Girardi, M. 2009, A\&A, 495, 15

Braglia, F. G., Pierini, D., Biviano, A., \& Böhringer, H. 2009, A\&A, 500, 947

Bridle, S., \& King L., 2007, New J. Phys., 9, 444

Brown, M. L., Taylor, A. N., Hambly, N. C., \& Dye, S. 2002, MNRAS, 333, 501

Bruzual, G., \& Charlot, S. 2003, MNRAS, 344, 1000

Cacciato, M., van den Bosch, F. C., More, S., Mo, H., \& Yang, X. 2013, MNRAS, 430, 767

Catelan, P., Kamionkowski, M., \& Blandford, R. D. 2001, MNRAS, 320, L7

Cava, A., Bettoni, D., Poggianti, B., et al. 2009, A\&A, 495, 707

Chisari, N. E., Mandelbaum, R., Straus, M. A., Huff, E., \& Bahcall, N. 2014, MNRAS, 445, 726

Christlein, D., \& Zabludoff, A. I. 2003, ApJ, 591, 764

Ciotti, L., \& Dutta, S. N. MNRAS, 270, 390

Colless, M., Dalton, G., Maddox, S., et al. 2001, MNRAS, 328, 1039

Colless, M., Peterson, B. A., Jackson, C., et al. 2003

[arXiv: astro-ph/0306581]

Cooray, A., \& Hu, W. 2001, ApJ, 554, 56

Crawford, S. M., Wirth, G. D., Bershady, M. A., \& Hon, K. 2011, ApJ, 741, 98

Czoke, O., Kneib, J.-P., Soucail, G., et al. 2001, A\&A, 372, 391

Danese, L., de Zotti, G., \& di Tullio, G. 1980, A\&A, 82, 322

de Jong, J. T. A., Verdoes Klijn, G. A., Kuijken, K. H., \& Valentijn, E. A. 2013,

Exper. Astron., 35, 25

Dekel, A. 1985, ApJ, 298, 461

Dietrich, J. P., Clowe, D. I., \& Soucail, G. 2002, A\&A, 394, 395

Djorgovski, S. 1983, ApJ, 274, L7

Dressler, A., \& Gunn, J. E. 1992, ApJS, 78, 1

Dressler, A., \& Schechtman, S. A. 1988, AJ, 95, 985

Drinkwater, M. J., Jurek, R. J., Blake, C., et al. 2010, MNRAS, 401, 1429

Duffy, A. R., Schaye, J., Kay, S. T., \& Dalla Vecchia, C. 2008, MNRAS, 390, L64

Durret, F., Felenbok, P., Lobo, C., \& Slezak, E. 1998, A\&AS, 129, 281

Ebeling, H., Ma, C. J., \& Barrett, E. 2014, ApJS, 211, 21

Ellingson, E., Yee, H. K. C., Abraham, R. G., et al. 1997, ApJS, 113, 1

Evrard, A. E., Bialek, J., Busha, M., et al. 2008, ApJ, 672, 122

Fadda, D., Girardi, M., Giuricin, G., Mardirossian, F., \& Mezzetti, M. 1996, ApJ, 473, 670

Faltenbacher, A., Li, C., Mao, S., et al. 2007a, ApJ, 662, L71

Faltenbacher, A., Hoffman, Y., Gottlöber, S., \& Yepes, G. 2007b, MNRAS, 376, 1327

Faltenbacher, A., Jing, Y. P., Li, C., et al. 2008, ApJ, 675, 146

Ferrari, C., Maurogordato, S., Cappi, A., \& Benoist, C. 2003, A\&A, 399, 813

Fisher, D., Fabricant, D., Franx, M., \& van Dokkum, P. 1998, 498, 195

Geller, M. J., Hwang, H. S., Diaferio, A., et al. 2014, ApJ, 783, 52

George, M. R., Leauthaud, A., Bundy, K., et al. 2012, ApJ, 757, 2

Girardi, M., Barrena, R., Boschin, W., \& Ellingson, E. 2008, A\&A, 491, 379

Godłowksi, W., Baier, F. W., \& MacGillivray, H. T. 1998, A\&A, 339, 709

Godłowski, W., Piwowarska, P., Panko, E., \& Flin, P. 2010, ApJ, 723, 985

Hall, A., \& Taylor, A. 2014, MNRAS, 403, L119

Hao, J., Koester, B. P., McKay, T. A., et al. 2009, ApJ, 702, 745

Hao, J., Kubo, J. M., Feldmann, R., et al. 2011, ApJ, 740, 39

Hawley, D. L., \& Peebles, P. J. E. 1975, AJ, 80, 477

Heymans, C., \& Heavens, A. 2003, MNRAS, 339, 711

Heymans, C., Brown, M., Heavens, A., et al. 2004, MNRAS, 347, 895

Heymans, C., Grocutt, E., Heavens, A., et al. 2013, MNRAS, 432, 2433

High, F. W., Stubbs, C. W., Rest, A., Stalder, B., \& Challis, P. 2009, AJ, 138, 110

Hill, J. M., Oegerle, W. R. 1993, AJ, 106, 831

Hirata, C. M., \& Seljak, U. 2004, Phys. Rev. D, 70, 063526

Hirata, C. M., Mandelbaum, R., Ishak, M., et al. 2007, MNRAS, 381, 1197

Hoekstra, H. 2007, MNRAS, 379, 317

Hoekstra, H., Franx, M., Kuijken, K., \& Squires, G. 1998, ApJ, 504, 636

Hoekstra, H., Mahdavi, A., Babul, A., \& Bildfell, C. 2012, MNRAS, 427, 1298

Huchra, J. P., Macri, L. M., Masters, K. L., et al. 2012, ApJS, 199, 26 
Hui, L., \& Zhang, J. 2002, [arXiv: astro-ph/0205512]

Hung, C.-L., \& Ebeling, H. 2012, MNRAS, 421, 3229

Hunter, J. D. 2007 Comput. Sci. Eng., 9, 90

Jäger, K., Ziegler, B. L., Böhm, A., et al. 2004, A\&A, 422, 907

Jee, M. J., Hoekstra, H., Mahdavi, A., \& Babul, A. 2014, ApJ, 783, 78

Joachimi, B., \& Bridle, S. L. 2010, A\&A, 523, A1

Joachimi, B., \& Schneider, P. 2008, A\&A, 488, 829

Joachimi, B., \& Schneider, P. 2010, A\&A, 517, A4

Joachimi, B., Mandelbaum, R., Abdalla, F. B., \& Bridle, S. L. 2011, A\&A, 527, A26

Joachimi, B., Semboloni, E., Bett, P. E., et al. 2013a, MNRAS, 431, 477

Joachimi, B., Semboloni, E., Hilbert, S., et al. 2013b, MNRAS, 436, 819

Kaiser, N. 1992, ApJ, 388, 272

Kaiser, N., Squires, G., \& Broadhurst, T. 1995, ApJ, 449, 460

Kelly, P. L., von der Linden, A., Applegate, D. E., et al. 2014, MNRAS, 439, 28

King, L. J. 2005, A\&A, 441, 47

King, L. J., \& Schneider, P. 2002, A\&A, 396, 411

Kirk, D., Bridle, S., \& Schneider, M. 2010, MNRAS, 408, 1502

Kirk, D., Rassat, A., Host, O., \& Bridle, S. L. 2012, MNRAS, 424, 1647

Kitching, T. D., Rowe, B., Gill, M., et al. 2013, ApJS, 205, 12

Kuijken, K. H. 2008, A\&A, 482, 1053

Liang, H., Lémonon, L, Valtchanov, I., Pierre, M., \& Soucail, G. 2000, A\&A, 363,440

Limber, D. N. 1953, ApJ, 117, 145

Lin, Y.-T., \& Mohr, J. J. 2004, ApJ, 617, 879

Luppino, G. A., \& Kaiser, N., 1997, ApJ, 475, 20

Magnier, E. A. \& Cuillandre, J.-C. 2004, PASP, 116, 449

Mahdavi, A., Hoekstra, H., Babul, A., et al. 2013, ApJ, 767, 116

Mamon, G. A., Biviano, A., \& Murante, G. 2010, A\&A, 520, A30

Mancone, C., \& González, A. 2012, PASP, 124, 606

Mandelbaum, R., Hirata, C. M., Seljak, U., et al. 2005, MNRAS, 361, 1287

Mandelbaum, R., Hirata, C. M., Ishak, M., Seljak, U., \& Brinkmann, J. 2006, MNRAS, 367, 611

Mandelbaum, R., Blake, C., Bridle, S. L., et al. 2011, MNRAS, 410, 844

Martini, P., Mulchaey, J. S., \& Kelson, D. 2007, ApJ, 664, 761

Massey, R., Heymans, C., Bergé, J., et al. 2007, MNRAS, 376, 13

Maurogordato, S., Cappi, A., Ferrari, C., et al. 2008, A\&A, 481, 593

Maurogordato, S., Sauvageot, J. L., Bourdin, H., et al. 2011, A\&A, 525, A79

Melchior, P., \& Viola, M. 2012, MNRAS, 424, 2757

Menanteau, F., Hughes, J. P., Sifón, C., et al. 2012, ApJ, 748, 7

Mercurio, A., Girardi, M., Boschin, W., Merluzzi, P., \& Busarello, G. 2003, A\&A, 397, 431

Miller, N. A., Owen, F. N., Hill, J. M., et al. 2004, ApJ, 613, 84

Miller, N. A., Oegerle, W. R., \& Hill, J. M. 2006, AJ, 131, 2426

Navarro, J. F., Frenk, C. S., \& White, S. D. M. 1995, MNRAS, 275, 720

Niederste-Ostholt, M., Strauss, M. A., Dong, F., Koester, B. P., \& McKay, T. A. 2010, MNRAS, 405, 2023

Oegerle, W. R., Hill, J. M., \& Fitchett, M. J. 1995, AJ, 110, 320

Okumura, T., Jin, Y. P., \& Cheng, L. 2009, ApJ, 694, 214

Owers, M. S., Nulsen, P. E. J., \& Couch, W. J. 2011, ApJ, 741, 122
Panko, E., Juszczyk, T., \& Flin, P. 2009, AJ, 138, 1709

Pen, U.-L., Lee, J., \& Seljak, U. 2000, ApJ, 543, L107

Peng, C. Y., Ho, L. C., Impey, C. D., \& Rix, H.-W. 2002, AJ, 124, 266

Pereira, M. J., \& Bryan, G. L. 2010, ApJ, 721, 939

Pereira, M. J., \& Kuin, J. R. 2005, ApJ, 627, L21

Pereira, M. J., Bryan, G. L., \& Gill, S. P. D. 2008, ApJ, 672, 825

Pérez, F., \& Granger, B. E. 2007, Comput. Sci. Eng., 9, 21

Pimbblet, K. A., Smail, I., Edge, A. C., et al. 2006, MNRAS, 366, 645

Pinkney, J., Roettiger, K., Burns, J. O., \& Bird, C. M. 1996, ApJS, 104, 1

Plionis, M., Benoist, C., Maurogordato, S., Ferrari, C., \& Basilakos, S. 2003, ApJ, 594, 144

Proust, D., Cuevas, H., Capelato, H. V., et al. 2000, A\&A, 355, 443

Rines, K., Geller, M. J., Diaferio, A., \& Kurtz, M. J. 2013, ApJ, 767, 15

Rood, H. J., \& Sastry, G. N. AJ, 77, 451

Sand, D. J., Graham, M. L., Bildfell, C., et al. 2012, ApJ, 746, 163

Sastry, G. N. 1968, PASP, 80, 252

Schlafly, E. F., \& Finkbeiner, D. P. 2011, ApJ, 737, 103

Schneider, M. D., \& Bridle, S. 2010, MNRAS, 402, 2127

Schneider, M. D., Cole, S., Frenk, C. S., et al. 2013, MNRAS, 433, 2727

Scoville, N., Abraham, R. G., Aussel, H., et al. 2007, ApJS, 172, 38

SDSS-III Collaboration 2012, ApJS, 203, 21

Semboloni, E., Hoekstra, H., Schaye, J., van Daalen, M. P., \& McCarthy, I. G. 2011, MNRAS, 417, 2020

Sérsic, J. L. 1968, Atlas de Galaxias Australes (Córdoba: Obs. Astron., Univ. Nac. Córdoba)

Sifón, C., Menanteau, F., Hasselfield, M., et al. 2013, ApJ, 722, 25

Skielboe, A., Wojtak, R., Pedersen, K., Rozo, E., \& Rykoff, E. S. 2012, ApJ, 758, L16

Smith, R. E., Peacock, J. A., Jenkins, A., et al. 2003, MNRAS, 341, 1311

Strutskie, M. F., Cutri R. M., Stiening, R., et al. 2006, AJ, 131, 1163

Tenneti, A., Mandelbaum, R., Di Matteo, T., Feng, Y., \& Khandai, N. 2014, MNRAS, 441, 470

Thompson, L. A. 1976, ApJ, 209, 22

Tinker, J. L., Robertson, B. E., Kravtsov, A. V. et al. 2010, ApJ, 724, 878

Trevese, D., Cirimele, G., \& Flin, P. 1992, AJ, 104, 935

Troxel, M. A., \& Ishak, M. 2014, Phys. Rev. D, 89, 063528

van Daalen, M. P., Schaye, J., Booth, C. M., \& Dalla Vecchia, C. 2011, MNRAS, 415, 3649

van der Burg, R. F. J., Muzzin, A., Hoekstra, H., et al. 2013, A\&A, 557, A15

van der Burg, R. F. J., Hoekstra, H., Muzzin, A., et al. 2015, A\&A, accepted [arXiv: 1412.2137]

van Kampen, E., \& Rhee, G. F. R. N. 1990, A\&A, 237, 283

Viola, M., Melchior, P., \& Bartelmann, M. 2011, MNRAS, 410, 2156

Yee, H. K. C., Ellingson, E., \& Carlberg, R. G. 1996, ApJS, 102, 269

Yee, H. K. C., Ellingson, E., Morris, S. L., Abraham, R. G., \& Carlberg, R. G. 1998, ApJS, 116, 211

York, D. G., Adelman, J., Anderson, J. E., Jr., et al. 2000, AJ, 120, 1579

Zhang, P. 2010a, ApJ, 720, 1090

Zhang, P. 2010b, MNRAS, 406, L95 
Table 2. Cluster sample, redshifts, and velocity dispersions.

\begin{tabular}{|c|c|c|c|c|c|c|c|c|c|}
\hline Cluster & $z$ & $N_{m}$ & $N_{200}$ & $\begin{array}{c}\sigma_{200} \\
\left(\mathrm{~km} \mathrm{~s}^{-1}\right)\end{array}$ & $\begin{array}{c}M_{200} \\
\left(10^{14} M_{\odot}\right)\end{array}$ & $\begin{array}{c}r_{200} \\
(\mathrm{Mpc})\end{array}$ & $\mathcal{S}_{\Delta}$ & $\begin{array}{c}\text { Main } \\
\text { sources }^{a}\end{array}$ & $\begin{array}{l}\text { Main NED } \\
\text { sources }^{b}\end{array}$ \\
\hline Abell 85 & 0.0555 & 284 & 248 & $967 \pm 55$ & $10.0 \pm 1.7$ & $2.03 \pm 0.12$ & $0.01_{-0.00}^{+0.00}(\mathrm{D})$ & 3 & 14 \\
\hline Abell 115 & 0.1930 & 73 & 73 & $1028 \pm 108$ & $11.2 \pm 3.5$ & $2.03 \pm 0.21$ & $0.24_{-0.07}^{+0.02}(\mathrm{R})$ & - & 1 \\
\hline Abell 119 & 0.0443 & 268 & 255 & $875 \pm 48$ & $7.5 \pm 1.2$ & $1.85 \pm 0.10$ & $<0.01(\mathrm{D})$ & 3 & 7 \\
\hline Abell 133 & 0.0558 & 62 & 59 & $791 \pm 79$ & $5.5 \pm 1.7$ & $1.66 \pm 0.17$ & $0.24_{-0.14}^{+0.03}(\mathrm{R})$ & - & 21 \\
\hline Abell $209^{c}$ & 0.2090 & 110 & 110 & $1170 \pm 99$ & $16.4 \pm 4.1$ & $2.28 \pm 0.19$ & $0.01_{-0.01}^{+0.00}(\mathrm{D})$ & - & 27 \\
\hline Abell $222^{c}$ & 0.2132 & 76 & 76 & $881 \pm 79$ & $7.0 \pm 1.9$ & $1.72 \pm 0.15$ & $0.30_{-0.04}^{+0.04}(\mathrm{R})$ & - & 11,33 \\
\hline Abell $223^{c}$ & 0.2076 & 64 & 64 & $910 \pm 80$ & $7.8 \pm 2.1$ & $1.78 \pm 0.16$ & $0.05_{-0.02}^{+0.02}(\mathrm{D})$ & - & 11,33 \\
\hline Abell 267 & 0.2291 & 219 & 156 & $1006 \pm 74$ & $10.3 \pm 2.3$ & $1.95 \pm 0.14$ & $<0.01(\mathrm{D})$ & 3,4 & - \\
\hline Abell 2670 & 0.0763 & 241 & 196 & $919 \pm 46$ & $8.5 \pm 1.3$ & $1.91 \pm 0.10$ & $<0.01(\mathrm{D})$ & 3 & 21 \\
\hline Abell 2703 & 0.1140 & 75 & 13 & $657 \pm 53$ & $3.1 \pm 0.8$ & $1.35 \pm 0.11$ & $0.17_{-0.05}^{+0.18}(\mathrm{R})$ & 3 & - \\
\hline Abell 383 & 0.1885 & 182 & 134 & $918 \pm 53$ & $8.1 \pm 1.4$ & $1.81 \pm 0.11$ & $<0.01(\mathrm{D})$ & - & 18 \\
\hline Abell 399 & 0.0718 & 250 & 229 & $1046 \pm 47$ & $12.5 \pm 1.7$ & $2.18 \pm 0.10$ & $<0.01(\mathrm{D})$ & 5 & 20 \\
\hline Abell 401 & 0.0735 & 104 & 83 & $933 \pm 81$ & $8.9 \pm 2.3$ & $1.94 \pm 0.17$ & $0.32_{-0.05}^{+0.19}(\mathrm{R})$ & - & 20 \\
\hline Abell 520 & 0.2007 & 153 & 127 & $1045 \pm 73$ & $11.8 \pm 2.5$ & $2.05 \pm 0.14$ & $0.27_{-0.11}^{+0.04}(\mathrm{R})$ & - & 19 \\
\hline Abell $521^{c}$ & 0.2469 & 95 & 95 & $1002 \pm 95$ & $10.1 \pm 2.9$ & $1.92 \pm 0.18$ & $0.06_{-0.02}^{+0.02}(\mathrm{D})$ & - & 16 \\
\hline Abell $545^{c}$ & 0.1577 & 80 & 80 & $1038 \pm 89$ & $11.8 \pm 3.0$ & $2.08 \pm 0.18$ & $0.07_{-0.00}^{+0.02}(\mathrm{R})$ & - & 2 \\
\hline Abell 553 & 0.0670 & 54 & 44 & $665 \pm 75$ & $3.3 \pm 1.1$ & $1.39 \pm 0.16$ & $0.01_{-0.00}^{+0.03}(\mathrm{D})$ & 5 & - \\
\hline Abell 586 & 0.1704 & 33 & 21 & $803 \pm 104$ & $5.4 \pm 2.1$ & $1.60 \pm 0.21$ & $0.28_{-0.07}^{+0.12}(\mathrm{R})$ & 3 & - \\
\hline Abell $644^{c}$ & 0.0696 & 31 & 31 & $625 \pm 96$ & $2.7 \pm 1.2$ & $1.31 \pm 0.20$ & $0.67_{-0.05}^{+0.11}(\mathrm{R})$ & - & 24 \\
\hline Abell 646 & 0.1266 & 259 & 69 & $707 \pm 66$ & $3.8 \pm 1.1$ & $1.44 \pm 0.13$ & $0.07_{-0.04}^{+0.22}(\mathrm{D})$ & 3,4 & - \\
\hline Abell 655 & 0.1271 & 306 & 109 & $938 \pm 57$ & $8.8 \pm 1.6$ & $1.91 \pm 0.12$ & $0.03_{-0.02}^{+0.01}(\mathrm{D})$ & 3,4 & - \\
\hline Abell 697 & 0.2821 & 215 & 106 & $1161 \pm 89$ & $15.4 \pm 3.6$ & $2.19 \pm 0.17$ & $0.44_{-0.02}^{+0.05}(\mathrm{R})$ & 3,4 & - \\
\hline Abell 754 & 0.0546 & 305 & 300 & $959 \pm 43$ & $9.8 \pm 1.3$ & $2.01 \pm 0.09$ & $0.01_{-0.00}^{+0.00}(\mathrm{D})$ & - & 8 \\
\hline Abell $780^{c}$ & 0.0547 & 33 & 33 & $822 \pm 113$ & $6.2 \pm 2.5$ & $1.73 \pm 0.24$ & $0.01_{-0.00}^{+0.01}(\mathrm{D})$ & - & 21 \\
\hline Abell 795 & 0.1385 & 166 & 117 & $768 \pm 59$ & $4.9 \pm 1.1$ & $1.56 \pm 0.12$ & $0.06_{-0.02}^{+0.07}(\mathrm{D})$ & $3,4,5$ & - \\
\hline Abell 851 & 0.4038 & 53 & 47 & $999 \pm 138$ & $9.3 \pm 3.8$ & $1.77 \pm 0.24$ & $0.01_{-0.01}^{+0.03}(\mathrm{D})$ & - & 3,12 \\
\hline Abell 959 & 0.2882 & 67 & 67 & $982 \pm 101$ & $9.4 \pm 2.9$ & $1.85 \pm 0.19$ & $0.08_{-0.03}^{+0.04}(\mathrm{D})$ & - & 5 \\
\hline Abell 961 & 0.1275 & 58 & 16 & $740 \pm 142$ & $4.4 \pm 2.5$ & $1.51 \pm 0.29$ & $0.84_{-0.13}^{+0.06}(\mathrm{R})$ & 3 & - \\
\hline Abell 963 & 0.2036 & 165 & 85 & $922 \pm 64$ & $8.1 \pm 1.7$ & $1.81 \pm 0.13$ & $0.17_{-0.06}^{+0.06}(\mathrm{R})$ & 3,4 & - \\
\hline Abell 990 & 0.1421 & 209 & 86 & $829 \pm 96$ & $6.1 \pm 2.1$ & $1.68 \pm 0.19$ & $0.74_{-0.05}^{+0.03}(\mathrm{R})$ & $3,4,5$ & - \\
\hline Abell 1033 & 0.1224 & 170 & 98 & $762 \pm 52$ & $4.8 \pm 1.0$ & $1.56 \pm 0.11$ & $0.59_{-0.21}^{+0.08}(\mathrm{R})$ & 3,4 & - \\
\hline Abell 1068 & 0.1393 & 104 & 32 & $740 \pm 160$ & $4.3 \pm 2.8$ & $1.50 \pm 0.32$ & $0.93_{-0.04}^{+0.04}(\mathrm{R})$ & 3,4 & - \\
\hline Abell 1132 & 0.1349 & 160 & 55 & $727 \pm 89$ & $4.1 \pm 1.5$ & $1.48 \pm 0.18$ & $0.13_{-0.05}^{+0.06}(\mathrm{R})$ & 3,4 & - \\
\hline Abell 1234 & 0.1638 & 54 & 30 & $513 \pm 86$ & $1.4 \pm 0.7$ & $1.03 \pm 0.17$ & $0.87_{-0.07}^{+0.06}(\mathrm{R})$ & 3,4 & - \\
\hline Abell 1246 & 0.1920 & 207 & 87 & $956 \pm 84$ & $9.1 \pm 2.4$ & $1.89 \pm 0.17$ & $0.01_{-0.00}^{+0.01}(\mathrm{D})$ & 3,4 & - \\
\hline Abell 1285 & 0.1078 & 77 & 51 & $826 \pm 90$ & $6.1 \pm 2.0$ & $1.70 \pm 0.19$ & $0.52_{-0.18}^{+0.08}(\mathrm{R})$ & 5 & - \\
\hline Abell 1361 & 0.1157 & 143 & 46 & $587 \pm 62$ & $2.2 \pm 0.7$ & $1.21 \pm 0.13$ & $0.39_{-0.07}^{+0.15}(\mathrm{R})$ & 3,4 & - \\
\hline Abell 1413 & 0.1418 & 124 & 65 & $881 \pm 81$ & $7.3 \pm 2.0$ & $1.78 \pm 0.16$ & $0.72_{-0.23}^{+0.07}(\mathrm{R})$ & 3,4 & - \\
\hline
\end{tabular}

Notes. Columns are: (1) cluster name; (2) cluster redshift; (3) number of members out to arbitrary radius; (4) number of members within $r_{200}$; (5) velocity dispersion of members within $r_{200}$; (6) total mass within $r_{200}$; (7) cluster radius $r_{200}$; (8) significance level of the DS test, the letter in parenthesis shows whether a cluster is classified as disturbed (D), relaxed (R), or intermediate (I). ${ }^{(a)}$ Numbers are: (1) WLTV; (2) CNOC; (3) SDSS; (4) HeCS; (5) MENeaCS-spec. See text for references. ${ }^{(b)}$ Catalogs extracted from NED that contribute significantly to each cluster. These are: (1) Barrena et al. (2007); (2) Barrena et al. (2011); (3) Belloni \& Roser (1996); (4) Boschin et al. (2004); (5) Boschin et al. (2009); (6) Braglia et al. (2009); (7) WINGS (Cava et al. 2009); (8) Christlein \& Zabludoff (2003); (9) 2dF (Colless et al. 2003); (10) Czoke et al. (2001); (11) Dietrich et al. (2002); (12) Dressler \& Gunn (1992); (13) WiggleZ (Drinkwater et al. 2010); (14) Durret et al. (1998); (15) Ebeling et al. (2014); (16) Ferrari et al. (2003); (17) Fisher et al. (1998); (18) Geller et al. (2014); (19) Girardi et al. (2008); (20) Hill \& Oegerle (1993); (21) 2MRS (Huchra et al. 2012); (22) Jäger et al. (2004); (23) Liang et al. (2000); (24) Martini et al. (2007); (25) Maurogordato et al. (2008); (26) Maurogordato et al. (2011); (27) Mercurio et al. (2003); (28) Miller et al. (2004); (29) Miller et al. (2006); (30) Oegerle et al. (1995); (31) Owers et al. (2011); (32) Pimbblet et al. (2006); (33) Proust et al. (2000). ${ }^{(c)}$ Spectroscopic members extend out to less than $0.8 r_{200 .}$ 
Table 2. continued.

\begin{tabular}{|c|c|c|c|c|c|c|c|c|c|}
\hline Cluster & $z$ & $N_{m}$ & $N_{200}$ & $\begin{array}{c}\sigma_{200} \\
\left(\mathrm{~km} \mathrm{~s}^{-1}\right)\end{array}$ & $\begin{array}{c}M_{200} \\
\left(10^{14} M_{\odot}\right)\end{array}$ & $\begin{array}{c}r_{200} \\
(\mathrm{Mpc}) \\
\end{array}$ & $\mathcal{S}_{\Delta}$ & $\begin{array}{c}\text { Main } \\
\text { sources }^{a}\end{array}$ & $\begin{array}{l}\text { Main NED } \\
\text { sources }^{b}\end{array}$ \\
\hline Abell 1650 & 0.0841 & 266 & 140 & $720 \pm 48$ & $4.1 \pm 0.8$ & $1.50 \pm 0.10$ & $0.99_{-0.02}^{+0.00}(\mathrm{R})$ & 3,5 & - \\
\hline Abell 1651 & 0.0847 & 214 & 138 & $903 \pm 51$ & $8.0 \pm 1.4$ & $1.87 \pm 0.11$ & $0.65_{-0.16}^{+0.04}(\mathrm{R})$ & - & 9,21 \\
\hline Abell 1689 & 0.1847 & 252 & 235 & $1429 \pm 81$ & $30.0 \pm 5.1$ & $2.82 \pm 0.16$ & $<0.01(\mathrm{D})$ & 3,4 & - \\
\hline Abell 1758 & 0.2772 & 133 & 34 & $744 \pm 107$ & $4.1 \pm 1.8$ & $1.41 \pm 0.20$ & $0.11_{-0.03}^{+0.06}(\mathrm{R})$ & 3,4 & - \\
\hline Abell 1763 & 0.2323 & 186 & 103 & $1130 \pm 81$ & $14.6 \pm 3.1$ & $2.18 \pm 0.16$ & $<0.01(\mathrm{D})$ & 3,4 & - \\
\hline Abell 1781 & 0.0622 & 54 & 16 & $419 \pm 93$ & $0.8 \pm 0.5$ & $0.88 \pm 0.19$ & $0.92_{-0.19}^{+0.02}(\mathrm{R})$ & 3 & - \\
\hline Abell 1795 & 0.0629 & 191 & 133 & $778 \pm 51$ & $5.2 \pm 1.0$ & $1.63 \pm 0.11$ & $0.26_{-0.09}^{+0.04}(\mathrm{R})$ & 3 & 21 \\
\hline Abell 1835 & 0.2506 & 195 & 41 & $762 \pm 106$ & $4.5 \pm 1.9$ & $1.46 \pm 0.20$ & $0.66_{-0.23}^{+0.06}(\mathrm{R})$ & 3,4 & - \\
\hline Abell 1914 & 0.1671 & 257 & 146 & $911 \pm 54$ & $7.9 \pm 1.4$ & $1.82 \pm 0.11$ & $0.86_{-0.05}^{+0.03}(\mathrm{R})$ & 3,4 & - \\
\hline Abell 1927 & 0.0953 & 138 & 58 & $725 \pm 58$ & $4.2 \pm 1.0$ & $1.50 \pm 0.12$ & $0.25_{-0.08}^{+0.02}(\mathrm{R})$ & 3,5 & - \\
\hline Abell 1942 & 0.2257 & 51 & 27 & $820 \pm 140$ & $5.6 \pm 2.9$ & $1.59 \pm 0.27$ & $0.04_{-0.01}^{+0.05}(\mathrm{D})$ & 3 & - \\
\hline Abell 1991 & 0.0587 & 175 & 99 & $553 \pm 45$ & $1.9 \pm 0.5$ & $1.17 \pm 0.10$ & $0.12_{-0.05}^{+0.09}(\mathrm{R})$ & 3,5 & - \\
\hline Abell 2029 & 0.0777 & 317 & 181 & $1152 \pm 58$ & $16.6 \pm 2.5$ & $2.39 \pm 0.12$ & $<0.01(\mathrm{D})$ & 3 & 21 \\
\hline Abell 2033 & 0.0796 & 190 & 88 & $911 \pm 69$ & $8.3 \pm 1.9$ & $1.89 \pm 0.14$ & $0.03_{-0.01}^{+0.03}(\mathrm{D})$ & 3 & 21 \\
\hline Abell 2050 & 0.1202 & 164 & 82 & $854 \pm 80$ & $6.7 \pm 1.9$ & $1.74 \pm 0.16$ & $0.34_{-0.03}^{+0.06}(\mathrm{R})$ & 3,4 & - \\
\hline Abell 2055 & 0.1028 & 154 & 69 & $697 \pm 64$ & $3.7 \pm 1.0$ & $1.44 \pm 0.13$ & $0.04_{-0.00}^{+0.02}(\mathrm{D})$ & 3,4 & 21 \\
\hline Abell 2064 & 0.0734 & 62 & 32 & $675 \pm 108$ & $3.4 \pm 1.6$ & $1.41 \pm 0.22$ & $0.40_{-0.05}^{+0.13}(\mathrm{R})$ & 3 & - \\
\hline Abell 2065 & 0.0725 & 219 & 164 & $1095 \pm 67$ & $14.3 \pm 2.6$ & $2.28 \pm 0.14$ & $0.03_{-0.01}^{+0.01}(\mathrm{D})$ & 3 & - \\
\hline Abell 2069 & 0.1139 & 331 & 146 & $966 \pm 63$ & $9.7 \pm 1.9$ & $1.98 \pm 0.13$ & $0.01_{-0.00}^{+0.00}(\mathrm{D})$ & 3,4 & - \\
\hline Abell 2104 & 0.1547 & 90 & 56 & $1081 \pm 126$ & $13.3 \pm 4.6$ & $2.17 \pm 0.25$ & $0.22_{-0.09}^{+0.09}(\mathrm{R})$ & - & 23 \\
\hline Abell 2111 & 0.2281 & 256 & 83 & $738 \pm 66$ & $4.1 \pm 1.1$ & $1.43 \pm 0.13$ & $0.46_{-0.04}^{+0.09}(\mathrm{R})$ & 3,4 & 29 \\
\hline Abell 2125 & 0.2466 & 141 & 55 & $857 \pm 122$ & $6.4 \pm 2.7$ & $1.65 \pm 0.23$ & $0.46_{-0.26}^{+0.01}(\mathrm{R})$ & - & 28 \\
\hline Abell 2142 & 0.0903 & 1052 & 731 & $1086 \pm 31$ & $13.9 \pm 1.2$ & $2.24 \pm 0.06$ & $0.01_{-0.00}^{+0.00}(\mathrm{D})$ & 3 & 31 \\
\hline Abell 2163 & 0.2004 & 309 & 290 & $1279 \pm 53$ & $21.5 \pm 2.7$ & $2.51 \pm 0.10$ & $0.03_{-0.01}^{+0.02}(\mathrm{D})$ & - & 25 \\
\hline Abell 2204 & 0.1507 & 100 & 15 & $782 \pm 278$ & $5.1 \pm 5.4$ & $1.58 \pm 0.56$ & $0.35_{-0.12}^{+0.33}(\mathrm{R})$ & - & 32 \\
\hline Abell 2219 & 0.2255 & 364 & 241 & $1189 \pm 65$ & $17.0 \pm 2.8$ & $2.30 \pm 0.13$ & $<0.01(\mathrm{D})$ & 3,4 & 4 \\
\hline Abell 2259 & 0.1602 & 158 & 77 & $901 \pm 70$ & $7.7 \pm 1.8$ & $1.80 \pm 0.14$ & $0.04_{-0.01}^{+0.12}(\mathrm{D})$ & 3,4 & - \\
\hline Abell 2261 & 0.2257 & 206 & 76 & $882 \pm 86$ & $7.0 \pm 2.0$ & $1.71 \pm 0.17$ & $0.03_{-0.02}^{+0.05}(\mathrm{D})$ & 3,4 & - \\
\hline Abell $2319^{c}$ & 0.0538 & 83 & 83 & $1101 \pm 99$ & $14.7 \pm 4.0$ & $2.31 \pm 0.21$ & $0.52_{-0.16}^{+0.07}(\mathrm{R})$ & - & 30 \\
\hline Abell 2390 & 0.2287 & 136 & 92 & $1120 \pm 113$ & $14.3 \pm 4.3$ & $2.17 \pm 0.22$ & $0.23_{-0.10}^{+0.01}(\mathrm{R})$ & 2 & - \\
\hline Abell 2409 & 0.1454 & 101 & 46 & $826 \pm 94$ & $6.0 \pm 2.0$ & $1.67 \pm 0.19$ & $0.16_{-0.03}^{+0.09}(\mathrm{R})$ & 3,5 & - \\
\hline Abell $2440^{c}$ & 0.0906 & 88 & 88 & $766 \pm 61$ & $4.9 \pm 1.2$ & $1.59 \pm 0.13$ & $0.13_{-0.02}^{+0.03}(\mathrm{R})$ & 3 & 26 \\
\hline Abell 2495 & 0.0790 & 98 & 46 & $631 \pm 55$ & $2.8 \pm 0.7$ & $1.32 \pm 0.12$ & $0.23_{-0.01}^{+0.06}(\mathrm{R})$ & 3,5 & - \\
\hline Abell 2537 & 0.2964 & 175 & 65 & $909 \pm 85$ & $7.4 \pm 2.1$ & $1.70 \pm 0.16$ & $0.05_{-0.01}^{+0.04}(\mathrm{D})$ & 3 & 6 \\
\hline Abell 2597 & 0.0829 & 39 & 17 & $682 \pm 131$ & $3.5 \pm 2.0$ & $1.42 \pm 0.27$ & $0.37_{-0.09}^{+0.15}(\mathrm{R})$ & - & 9,13 \\
\hline CL 0024.0+1652 & 0.3948 & 229 & 131 & $757 \pm 48$ & $4.1 \pm 0.8$ & $1.35 \pm 0.09$ & $<0.01(\mathrm{D})$ & - & 10 \\
\hline MACS J0717.5+3745 & 0.5436 & 468 & 215 & $1370 \pm 79$ & $22.0 \pm 3.8$ & $2.24 \pm 0.13$ & $<0.01(\mathrm{D})$ & - & 15 \\
\hline MKW3S & 0.0444 & 125 & 82 & $592 \pm 49$ & $2.3 \pm 0.6$ & $1.25 \pm 0.11$ & $0.83_{-0.11}^{+0.06}(\mathrm{R})$ & 3 & - \\
\hline MS 0015.9+1609 & 0.5475 & 232 & 122 & $1330 \pm 115$ & $20.0 \pm 5.2$ & $2.17 \pm 0.19$ & $0.32_{-0.18}^{+0.10}(\mathrm{R})$ & 1,2 & - \\
\hline MS 0440.5+0204 & 0.1962 & 51 & 35 & $742 \pm 103$ & $4.3 \pm 1.8$ & $1.46 \pm 0.20$ & $0.26_{-0.13}^{+0.14}(\mathrm{R})$ & 2 & - \\
\hline MS 0451.6-0305 & 0.5382 & 247 & 200 & $1252 \pm 55$ & $16.8 \pm 2.2$ & $2.05 \pm 0.09$ & $<0.01(\mathrm{D})$ & 1,2 & - \\
\hline MS 1008.1-1224 & 0.3077 & 86 & 85 & $1028 \pm 92$ & $10.6 \pm 2.8$ & $1.91 \pm 0.17$ & $0.59_{-0.11}^{+0.07}(\mathrm{R})$ & 2 & 22 \\
\hline MS 1224.7+2007 & 0.3258 & 33 & 29 & $790 \pm 95$ & $4.8 \pm 1.7$ & $1.46 \pm 0.17$ & $0.57_{-0.07}^{+0.23}(\mathrm{R})$ & 2,3 & - \\
\hline MS $1231.3+1542$ & 0.2347 & 84 & 65 & $710 \pm 57$ & $3.7 \pm 0.9$ & $1.38 \pm 0.11$ & $0.87_{-0.03}^{+0.03}(\mathrm{R})$ & 2,3 & - \\
\hline MS 1358.4+6245 & 0.3289 & 189 & 152 & $1021 \pm 56$ & $10.3 \pm 1.7$ & $1.88 \pm 0.10$ & $0.01_{-0.00}^{+0.01}(\mathrm{D})$ & 2 & 17 \\
\hline MS $1455.0+2232$ & 0.2565 & 57 & 57 & $928 \pm 111$ & $8.0 \pm 2.9$ & $1.77 \pm 0.21$ & $0.58_{-0.24}^{+0.07}(\mathrm{R})$ & 2,3 & - \\
\hline MS $1512.4+3647$ & 0.3719 & 30 & 29 & $960 \pm 170$ & $8.4 \pm 4.4$ & $1.73 \pm 0.30$ & $0.04_{-0.00}^{+0.03}(\mathrm{D})$ & 2 & - \\
\hline MS $1621.5+2640$ & 0.4254 & 70 & 41 & $724 \pm 82$ & $3.5 \pm 1.2$ & $1.27 \pm 0.14$ & $0.17_{-0.04}^{+0.20}(\mathrm{R})$ & 2 & - \\
\hline
\end{tabular}


Table 2. continued.

\begin{tabular}{lccccccccc}
\hline \hline Cluster & $z$ & $N_{m}$ & $N_{200}$ & $\begin{array}{c}\sigma_{200} \\
\left(\mathrm{~km} \mathrm{~s}^{-1}\right)\end{array}$ & $\begin{array}{c}M_{200} \\
\left(10^{14} M_{\odot}\right)\end{array}$ & $\begin{array}{c}r_{200} \\
(\mathrm{Mpc})\end{array}$ & $\begin{array}{c}\mathcal{S}_{\Delta} \\
\begin{array}{c}\text { Main } \\
\text { sources }^{a}\end{array}\end{array} \begin{array}{c}\text { Main NED } \\
\text { sources }^{b}\end{array}$ \\
\hline RX J0736.6+3924 & 0.1179 & 62 & 28 & $432 \pm 64$ & $0.9 \pm 0.4$ & $0.89 \pm 0.13$ & $0.52_{-0.03}^{+0.12}(\mathrm{R})$ & 3,5 & - \\
ZwCl 0628.1+2502 & 0.0814 & 72 & 66 & $843 \pm 96$ & $6.6 \pm 2.2$ & $1.75 \pm 0.20$ & $0.04_{-0.01}^{+0.02}(\mathrm{D})$ & 5 & - \\
ZwCl 1023.3+1257 & 0.1425 & 84 & 24 & $622 \pm 108$ & $2.6 \pm 1.3$ & $1.26 \pm 0.22$ & $0.79_{-0.17}^{+0.17}(\mathrm{R})$ & 3,4 & - \\
ZwCl 1215.1+0400 & 0.0773 & 183 & 107 & $902 \pm 65$ & $8.0 \pm 1.7$ & $1.88 \pm 0.14$ & $0.29_{-0.04}^{+0.02}(\mathrm{R})$ & 3 & - \\
\hline
\end{tabular}

